\title{
Monte Carlo simulation of coagulation in discrete particle-size distributions. Part 2. Interparticle forces and the quasi-stationary equilibrium hypothesis
}

\author{
By I. A. VALIOULIS, E. J. LIST AND H. J. PEARSON \\ W. M. Keck Laboratory of Hydraulics and Water Resources, California Institute of \\ Technology, Pasadena, California 91125
}

(Received 8 August 1983 and in revised form 20 February 1984)

\begin{abstract}
Hunt (1982) and Friedlander $(1960 a, b)$ used dimensional analysis to derive expressions for the steady-state particle-size distribution in aerosols and hydrosols. Their results were supported by the Monte Carlo simulation of a non-interacting coagulating population of suspended spherical particles developed by Pearson, Valioulis \& List (1984). Here the realism of the Monte Carlo simulation is improved by accounting for the modification to the coagulation rate caused by van der Waals', electrostatic and hydrodynamic forces acting between particles. The results indicate that the major hypothesis underlying the dimensional reasoning, that is, collisions between particles of similar size are most important in determining the shape of the particle size distribution, is valid only for shear-induced coagulation. It is shown that dimensional analysis cannot, in general, be used to predict equilibrium particle-size distributions, mainly because of the strong dependence of the interparticle force on the absolute and relative size of the interacting particles.
\end{abstract}

\section{The quasi-stationary particle-size distribution}

Reasoning on dimensional grounds, Friedlander $(1960 a, b)$ and Hunt (1982) derived expressions for the steady-state evolution of the size distribution $n(v)$ of coagulating particles. $n(v)$ is defined by

$$
\mathrm{d} N=n(v) \mathrm{d} v,
$$

where $\mathrm{d} N$ is the number of particles with volumes in the range $v$ to $v+\mathrm{d} v$ per unit volume of fluid, so that $n(v)$ is the number density of particles in $v$-space.

The underlying idea was inspired from Kolmogorov's equilibrium theory of turbulence (see Monin \& Yaglom 1975). Friedlander assumed that a state of dynamic equilibrium would exist between production, coagulation and loss through sedimentation of particles in atmospheric aerosols. He hoped that there exist size ranges where only one of the coagulation mechanisms listed in table 1 is important, in which case the size distribution in some subrange would depend only on the particle volume $v$, the constant particle volume flux $E$, per unit volume of fluid, through the size distribution, and a dimensional parameter $\left(K_{\mathrm{B}}, K_{\mathrm{sh}}=G\right.$ or $K_{\mathrm{sh}}=(\varepsilon / \nu)^{\frac{1}{2}}$ and $\left.K_{\mathrm{ds}}\right)$ characterizing the dominant coagulation mechanism (see table 1). Hunt extended 


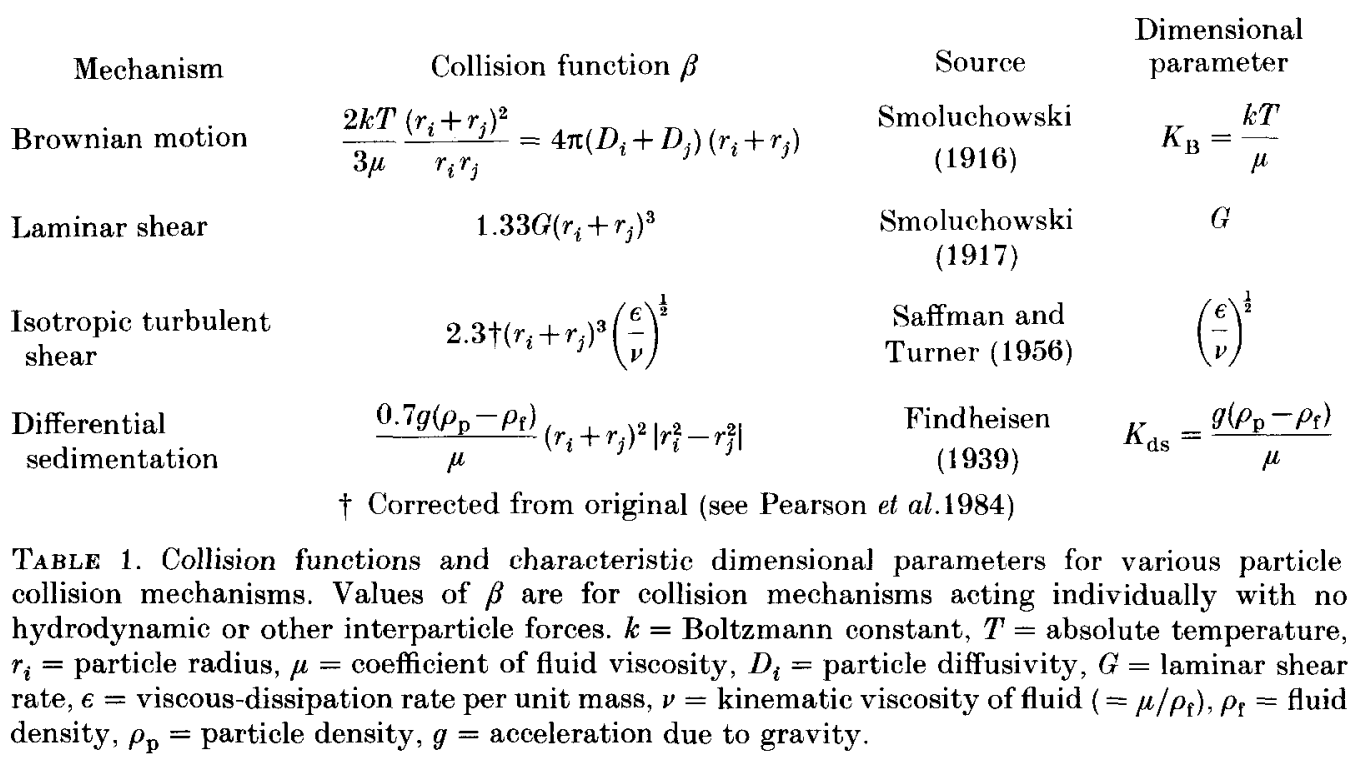

Friedlander's ideas to hydrosols and included a shearing- and differential-settlingdominated subrange. His expressions are

$$
\begin{aligned}
& n(v)=a_{\mathrm{B}}\left(\frac{E}{K_{\mathrm{B}}}\right)^{\frac{1}{2}} v^{-\frac{3}{2}}, \\
& n(v)=a_{\mathrm{sh}}\left(\frac{E}{K_{\mathrm{sh}}}\right)^{\frac{1}{2}} v^{-2}, \\
& n(v)=a_{\mathrm{ds}}\left(\frac{E}{K_{\mathrm{ds}}}\right)^{\frac{1}{2}} v^{-\frac{13}{6}},
\end{aligned}
$$

where $a_{\mathrm{B}}, a_{\mathrm{sh}}$ and $a_{\mathrm{ds}}$ are dimensionless constants.

Jeffrey (1981) offered a new derivation of Hunt's results which clarifies the assumptions involved in the dimensional arguments. The change with time of the particle-size distribution $n(v)$ is given by the general dynamic equation

$$
\begin{aligned}
\frac{\partial n(v)}{\partial t}=l(v)+\frac{1}{2} \int_{0}^{v} \beta\left(v^{\prime}, v-v^{\prime}\right) n\left(v^{\prime}\right) & n\left(v-v^{\prime}\right) \mathrm{d} v^{\prime} \\
& -\int_{0}^{\infty} \beta\left(v, v^{\prime}\right) n(v) n\left(v^{\prime}\right) \mathrm{d} v^{\prime}+w(v) \frac{\partial n(v)}{\partial z},
\end{aligned}
$$

where $\beta\left(v, v^{\prime}\right)$ is the collision function which represents the geometry and dynamies of the collision mechanism, $l(v)$ is a source of particles (through condensation, for example) and $w(v) \partial n(v) / \partial z$ is a particle sink resulting from particles sedimenting in the $z$-direction at their Stokes settling velocity $w(v)$. For homogeneous particle systems and for size ranges where the source term is negligible, the steady-state form of $(1.5)$ is

$$
\frac{1}{2} \int_{0}^{v} \beta\left(v^{\prime}, v-v^{\prime}\right) n\left(v^{\prime}\right) n\left(v-v^{\prime}\right) \mathrm{d} v^{\prime}=\int_{0}^{\infty} \beta\left(v, v^{\prime}\right) n(v) n\left(v^{\prime}\right) \mathrm{d} v^{\prime} .
$$

The integral on the left-hand side of (1.6) represents the rate of gain of particles of volume $v$ by coagulation of pairs of smaller particles, conserving volume; the integral 
on the right-hand side represents the flux of particles out of the size range $(v, v+\mathrm{d} v)$ due to their coagulation with particles of all sizes. Derivation of Hunt's expressions proceeds (Jeffrey 1981) under the assumption that collisions between particles of similar size contribute mostly to the r.h.s. term of (1.6). Jeffrey approximates

$$
\int_{0}^{\infty} \beta\left(v, v^{\prime}\right) n(v) n\left(v^{\prime}\right) \mathrm{d} v^{\prime} \approx \beta(v, v) n^{2}(v) v
$$

which, if multiplied by $v^{2}$ to convert from number-density flux to volume flux, is precisely the flux $E$ of particle volume through the size space. The general expression then follows:

$$
n(v) \sim\left(\frac{E}{\beta}\right)^{\frac{1}{2}} v^{-\frac{3}{2}}
$$

The collision function $\beta\left(v, v^{\prime}\right)$ is the probability that two particles of sizes $v$ and $v^{\prime}$ will collide in unit time. This probability is equal to the common volume two particles sweep per unit time under the influence of one or more physical mechanisms in a unit volume of fluid. If non-interference of the different coagulation mechanisms is assumed, then subranges exist where a sole mechanism dominates and $\beta\left(v, v^{\prime}\right)$ is given by the expressions listed in table 1; from (1.8) Hunt's expressions then follow.

It is clear that two assumptions are needed for the quasi-stationary distributions to be valid:

1. collisions between particles of similar size are more important, or, equivalently, that there in non-interference of particles of a size characteristic of one collision mechanism with those of another;

2. an equilibrium size distribution is established.

The latter assumption can be justified from the regularities observed in the size distributions of both atmospheric aerosols (Friedlander 1960 $a, b$ ) and hydrosols (Faisst 1976).

\section{Verification of the theory}

Hunt (1982) studied the coagulation of solid particles (three types of small clay particles and finely divided crystalline silica) in artificial seawater in the laminar shear generated between two rotating coaxial cylinders when the outer one was rotated. Some of his results support the predictions of the theory for Brownian-motion- and laminar-shear-induced coagulation, but none of the steady-state size distributions attained in the experiments had size regimes exhibiting the power-law behaviour of both the coagulation mechanisms. Settling of particles caused Hunt's systems to be in a quasi-dynamic steady state: the size distributions obtained were decreasing in magnitude while remaining similar in shape as the time progressed. Also, the dimensionless parameters $a_{\mathrm{B}}$ and $a_{\mathrm{sh}}$ appearing in (1.2) and (1.3) were not the same for the different suspensions studied. Hunt attributed this variation to properties of the suspensions which modified the coagulation rate.

In Part 1 (Pearson, Valioulis \& List 1984) we developed a method for Monte Carlo simulation of the evolution of a coagulating suspension. Spherical particles move in a cubical box or 'control' volume under the influence of Brownian motion and/or fluid shear. Hydrodynamic forces and colloidal forces are ignored so that particles move on straight paths. Particles in suspension have unit volume $v_{0}$, or integral multiples $v_{i}=i v_{0}$ of the unit volume. All lengths and times in the computer model are expressed as multiples of the radius of the unit particle and the time step $\Delta t$, both set equal to unity in the simulation. Colliding particles coagulate to form a larger, 
still-spherical particle, conserving volume. The model employs periodic boundary conditions which allow an infinite homogeneous system to be simulated approximately by a finite volume. A system in dynamic equilibrium is successfully modelled by using the following technique. A fixed number $N_{\mathrm{c}}$ of particles of unit volume are added to the population at random each time step, and any particles that have reached a preset maximum volume $v_{\max }$ are removed. The addition of small particles is a crude representation of the particle volume flux $E$ per unit volume of fluid, where $E=N_{\mathrm{c}} v_{0} / V \Delta t$, into the size range from coagulation of particles smaller than $v_{0}$. The removal of particles larger than $v_{\max }$ represents the physical loss of particles from the box by sedimentation or vertical concentration gradients. This procedure is consistent with the first hypothesis of the theory and is justified a posteriori by the success of the simulation on reproducing Hunt's (1982) dimensional results for Brownian motion, laminar shear- and isotropic turbulent shear-induced coagulation. In Part 1 we concluded that the final steady-state size distributions attained in our computer 'experiments' were insensitive to the size range covered by the simulation. However, as in Hunt's experiments, no one single simulation gave a size distribution having both Brownian-motion- and shear-coagulation-dominated regimes.

Our computer program, operating in a different mode, allows also the direct measurement of the collision function. On collision, particles are not coagulated but one of them simply repositioned so as to avoid repeated collisions of the same particle pair. In this manner the analytic estimates for the collision function for laminar shear and isotropic turbulent shear were reproduced.

The present study is a sequel to the Part 1 , and is an attempt to improve the realism of the results contained therein by accounting for the modifications to the coagulation rate caused by hydrodynamic, van der Waals and electrostatic forces acting between the approaching particles. Coagulation induced by differential sedimentation is also modelled, and the validity of Hunt's (1982) dimensional arguments is reexamined in the light of the results of the simulations performed in this study.

\section{Brownian diffusion}

\subsection{Particle interactions}

When forces act between two diffusing particles their collision rate, per unit volume of fluid, becomes

$$
\frac{2}{3} \frac{k T}{\mu} \frac{\left(r_{1}+r_{2}\right)^{2}}{r_{1} r_{2}} E_{\mathrm{B}}\left(r_{1}, r_{2}\right)
$$

where $E_{\mathrm{B}}\left(r_{1}, r_{2}\right)$ is a collision efficiency or modification to the collision rate, resulting from the interacting forces. Valioulis \& List (1983), following Spielman's (1970) method, computed the collision efficiency $E_{\mathrm{B}}$ for two spherical particles in Brownian diffusion assuming Stokes' flow and accounting for van der Waals' and electrostatic forces. Using Einstein's (1926) ingenious argument, they assumed a hypothetical equilibrium in an unbounded system of particles of two sizes, where the mean radial density flux of particles 2 relative to particle 1 due to Brownian motion is balanced by an advective flux which arises from an arbitrary conservative force $F$ acting between the particles. Considering only binary particle encounters and neglecting inertial effects so that the two fluxes become superposable (Batchelor 1976), it is then assumed that the expression for the relative particle diffusivity derived, $D=b k T$, is valid even when the force $F$ is removed. The relative mobility $b$ can be computed from the exact solution of Stokes' equations for two spheres moving along their line of centres obtained by Stimson \& Jeffrey (1926). Valioulis \& List (1983) solved the 


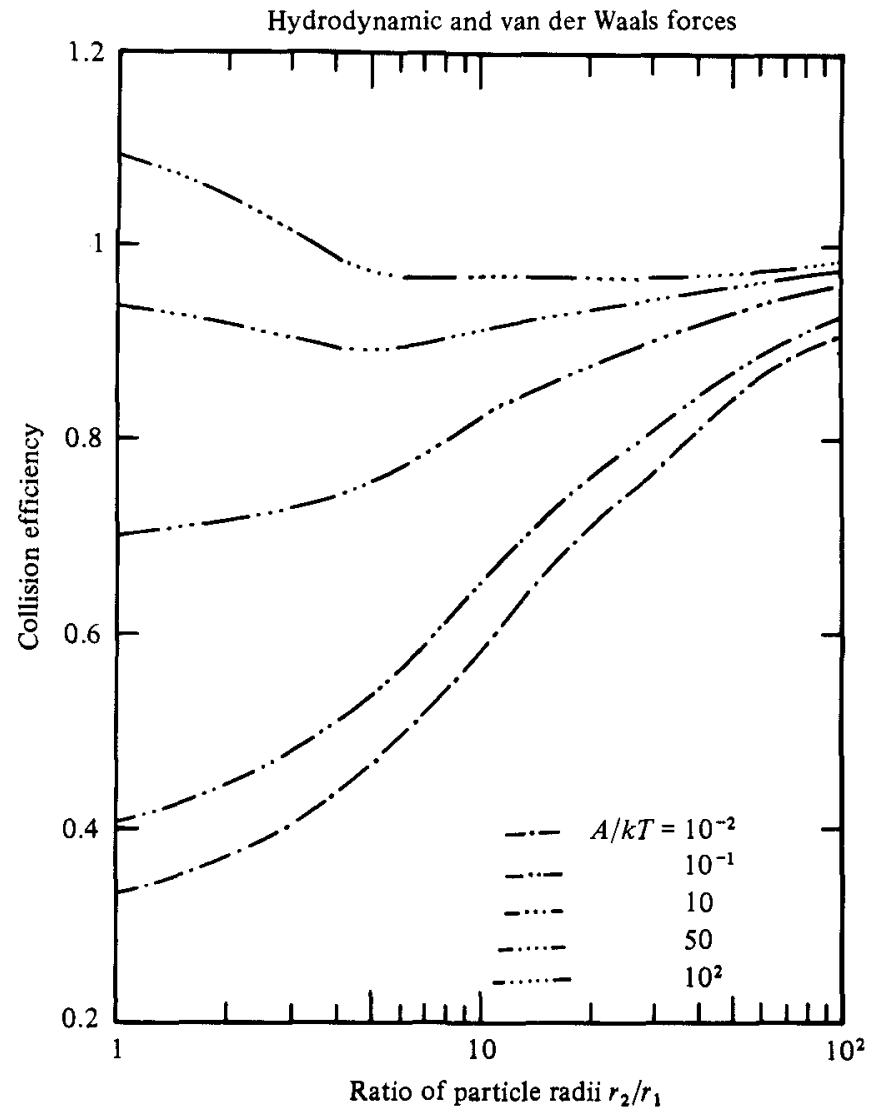

FIGURE 1. Collision efficiencies of particles with various relative sizes and for various values of $A / k T$ when van der Waals and hydrodynamic forces operate. The collision efficiencies were computed assuming that $\lambda / r_{1}=0.1$, where $\lambda$ is the London wavelength, typically about $0.1 \mu \mathrm{m}$.

diffusion equation numerically, including the van der Waals' attractive force as given by the expression of Schenkel \& Kitchener (1960) which includes microwave retardation effects, important for solid particles in water. Figure 1 illustrates the functional dependence of the collision efficiency on the relative size of the interacting particles for several values of the Hamaker constant $A$. It is seen that the combined action of hydrodynamic and van der Waals' forces reduces the collision rate, the effect being more pronounced for particles of similar size. For $r_{2} / r_{1}=1$ the collision efficiencies computed are slightly smaller than the ones obtained by Spielman (1970) and Honig, Roebersen \& Wiersma (1971), who did not account for retardation effects.

Valioulis \& List (1983) also incorporated double-layer forces in their computations, assuming that the two particles carry the same negative charge - since most dispersed particles in natural waters are negatively charged - and that the particle surface charge density rather than their surface potential remains constant during the Brownian encounter. The latter assumption is justified by the arguments of Frens \& Overbeek (1971), who showed that the timescale of the Brownian interaction of two particles (of the order of $10^{-7} \mathrm{~s}$ ) is too short for electrochemical equilibrium to be restored. The salient feature of the results is that two coagulation regimes can be identified: the 'rapid' coagulation regime, where the particle behaviour is not influenced by electrostatic interactions, and the 'slow' coagulation regime, where the 
suspension is stable for all practical purposes. The transition from a kinetically stable (no significant change in the number density of the particles during the observation time) to an unstable state of the dispersion is abrupt, so a quantitative criterion of coagulation (or stability) can exist. Using the results of Honig \& Mull (1971), Valioulis \& List (1983) obtained the following criterion for the onset of coagulation for suspended particles in water (assumed to be equivalent to a monovalent symmetrical electrolyte with the same ionic strength) at $20^{\circ} \mathrm{C}$ :

$$
\Phi \geqslant 1.29 \times 10^{-6} \frac{\sigma^{\frac{4}{3}}}{A^{\frac{2}{3}}}
$$

valid for $A \sigma<2 \times 10^{-26}$, where $\sigma$ is the particle surface charge density (in $\mathrm{C} / \mathrm{cm}^{2}$ ), $\Phi$ the ionic strength of electrolyte (in mol/litre) and $A$ the Hamaker constant (in $J$ ).

\subsection{Computer simulation}

For Brownian-induced coagulation in the presence of van der Waals' forces and hydrodynamic interactions, the functional dependence of the collision efficiency on the relative size of the interacting particles (see figure 1) suggests that the first assumption in the equilibrium theory is invalid.

The computer simulation of Part 1 is used to investigate the dependence of the steady-state size distribution on the externally imposed conditions - in particular, the particle-size range covered in any computer run. The collision function $\beta$ depends only on the relative size of the interacting particles; the collision efficiency $E_{\mathrm{B}}$ depends both on the relative and the absolute (when retardation effects are included) size of the interacting particles. The collision rate of two particles with radii $r_{1}$ and $r_{2}$, per unit time and per volume $V$ of fluid, under the influence of hydrodynamic and van der Waals' forces, can be set equal to the collision rate of two non-interacting particles with radii $t_{1}$ and $t_{2}$, per volume $V_{t}$ of fluid and per unit time:

$$
\frac{2}{3} \frac{k T}{\mu} \frac{\left(r_{1}+r_{2}\right)^{2}}{r_{1} r_{2}} \frac{E_{\mathrm{B}}}{V}=\frac{2}{3} \frac{k T}{\mu} \frac{\left(t_{1}+t_{2}\right)^{2}}{t_{1} t_{2}} \frac{1}{V_{t}}
$$

Solving equation (3.2) for $t_{2} / t_{1}$, we obtain

$$
\frac{t_{2}}{t_{1}}=-1+\frac{1}{2} \frac{\left(1+r_{2} / r_{1}\right)^{2}}{r_{2} / r_{1}} \frac{E_{\mathrm{B}}}{E_{1}}+\frac{1}{2}\left(1+\frac{r_{2}}{r_{1}}\right)\left\{\frac{E_{\mathrm{B}}}{E_{1}} \frac{r_{1}}{r_{2}}\left[\frac{\left(1+r_{2} / r_{1}\right)^{2}}{r_{2} / r_{1}} \frac{E_{\mathrm{B}}}{E_{1}}-4\right]\right\}^{\frac{1}{2}},
$$

where we have put $V=E_{1} V_{t} ; E_{1}$ is the collision efficiency for $r_{2} / r_{1}=1$ and is introduced so that (3.2) has real roots. For $r_{2} / r_{1}=1(3.3)$ gives $t_{2} / t_{1}=1$. Thus the collision rate in monodisperse non-interacting system of particles, per volume $E_{1} V_{t}$ of fluid, is equal to the collision rate, per volume $V$ of fluid, in a system of the same number of particles of equal size between which hydrodynamic and van der Waals' forces act (hereinafter referred to as the realistic system). Equation (3.3) maps the realistic system of particles of all sizes onto a non-interacting particle system; to every particle with radius $r$ ( $r$-particle) in the realistic system corresponds a particle with radius $t$ ( $t$-particle) in the non-interacting system. When two $t$-particles collide the corresponding $r$-particles are coalesced to form a new $r$-particle, conserving volume, and the radius of the new $t$-particle is calculated from (3.3). The method for generating displacements at each step and updating their positions is described in detail in Part 1. The initial volume concentration of suspended particles used in the simulations ranges from 0.1 to $1 \%$; such a high concentration is necessary in order to achieve results in reasonable computation times.

Figure 2 shows the time development of the normalized particle-size distribution 


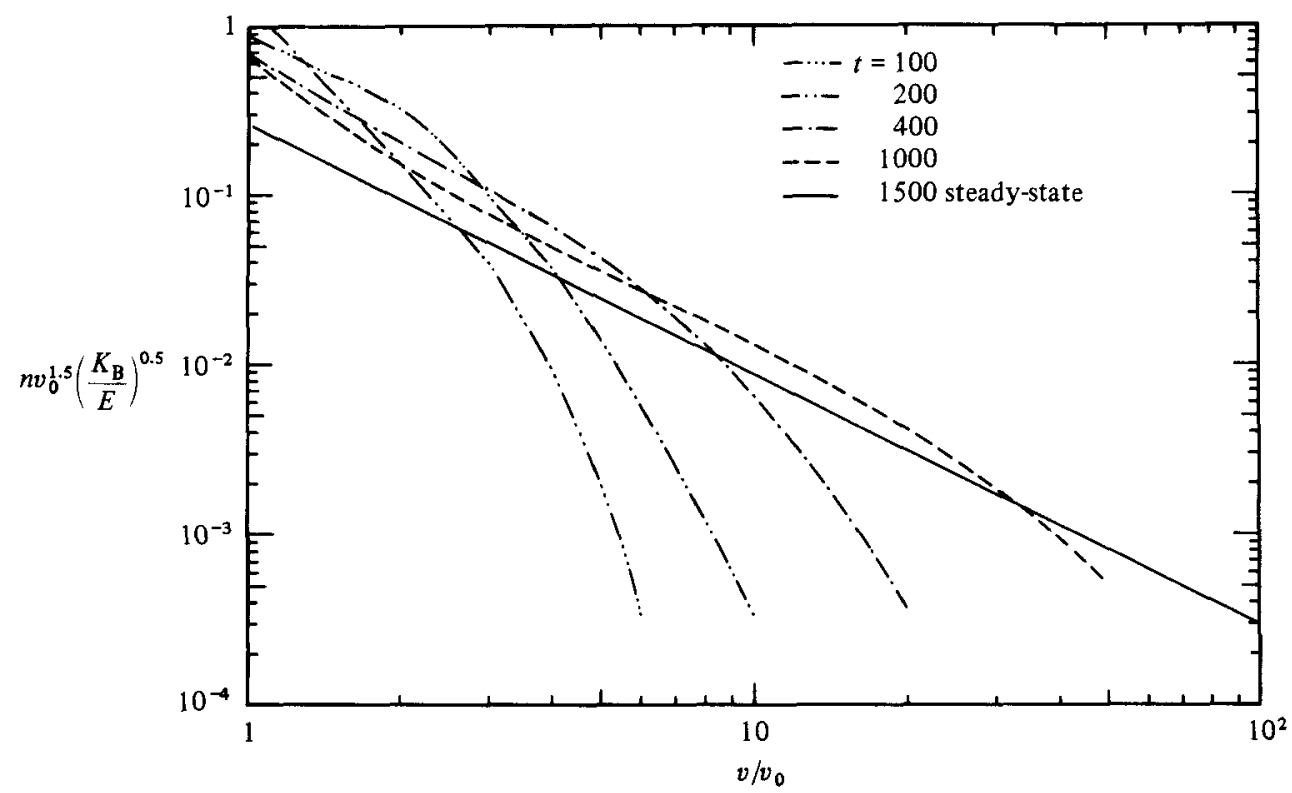

Figure 2. Evolution of the normalized size distribution for Brownian motion; $A / k T=1$, $\lambda / r_{1}=0.1, D_{0}=0.222, E=1.4 \times 10^{-5}, i_{\max }=125$.

of a population of particles undergoing Brownian-induced coagulation. The suspension is initially monodisperse and has a volume concentration of $\phi=0.57 \%$. The curves shown are smoothed approximations to ensemble averages of actual data points from five simulation runs. The data in the small size range attain a slope of about $-\frac{3}{2}$ once particles tenfold in volume are created. The level of the distribution declines then gradually until, after about 1200 time steps, a dynamic equilibrium is reached; this occurs when the first large particle is physically removed from the 'control' volume.

Dimensional analysis suggests that the value of the dimensionless group $D_{0} \Delta t / r_{0}^{2}$ should remain constant when the results are interpreted in the physical context. To illustrate, consider an aerosol particle with radius $1 \mu \mathrm{m}$ and diffusivity $1.3 \times 10^{-7} \mathrm{~cm}^{2} / \mathrm{s}$. Since $D_{0}=0.222, r_{0}=1$ and $\Delta t=1$ in the simulation, for a micron-sized particle $1 \mathrm{~s}$ of real time corresponds to about 58 time steps. Thus, for the volume concentration used here, the growth of the population of suspended particles examined is very rapid.

The series of simulation runs shown in figures 3 and 4 illustrate the effect that the ratio $i_{\max }=v_{\max } / v_{0}$ (i.e. the size range covered by the simulation) has on the final steady-state size distributions; $v_{0}$ is the unit particle volume and $v_{\max }$ the volume of the largest particle allowed to remain in the system. All simulation runs were started with a monodisperse population of particles. In both figures three runs with $v_{\max } / v_{0}=27,125$ and 512 are shown. For the simulations in figure 3 the Hamaker group $A / k T$ is 1 , and for those in figure 4 it is 0.01 (it thus covers the range of Hamaker constants found in natural waters). The data points shown are averaged over 2000 time steps after the first large particle was lost from the 'control' volume. This is necessary because of the small number of particles involved in the simulation. The data points, when non-dimensionalized according to (1.2) and plotted logarithmically against particle volume (non-dimensionalized with the unit particle volume), collapse onto a slope of $-\frac{3}{2}$. The level of the distributions as determined by the intercept of 


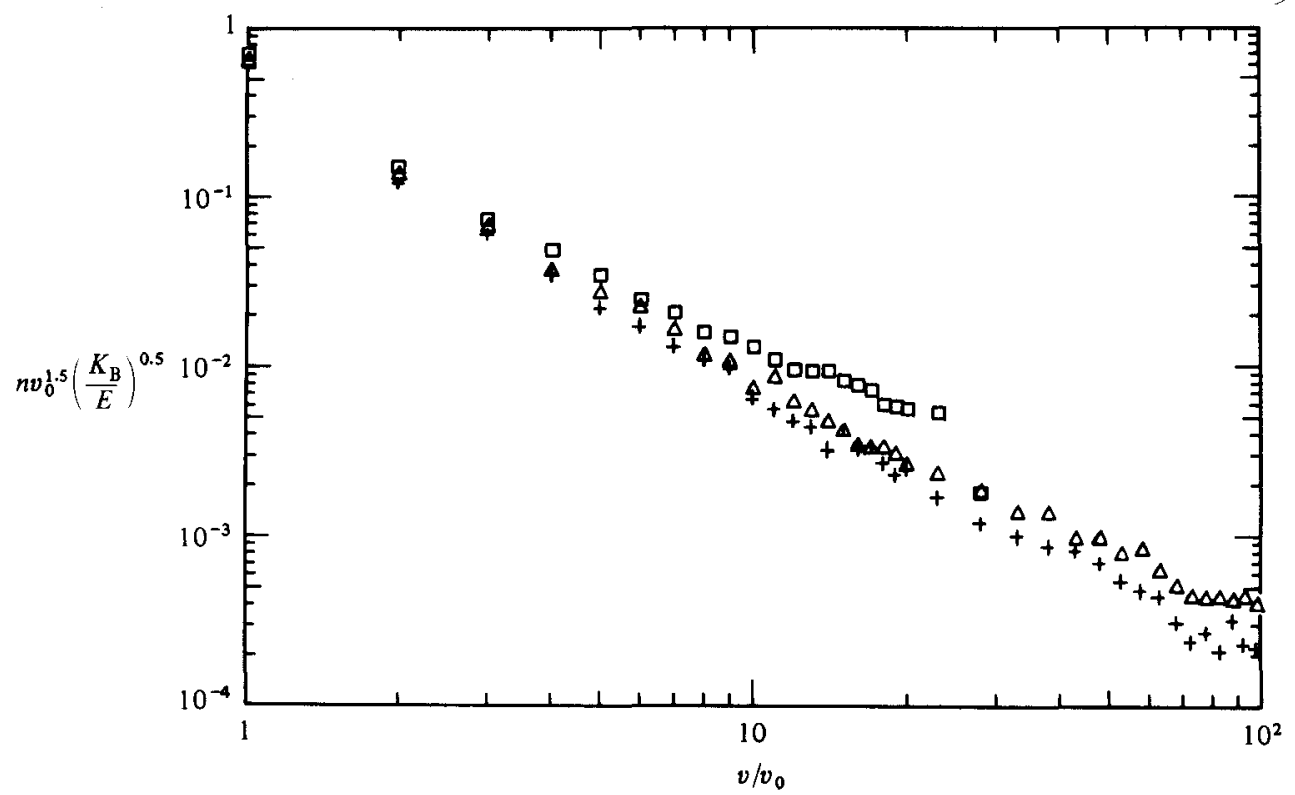

FigURE 3. Comparison of the steady-state non-dimensional size distribution for Brownian motion for different $i_{\max }$ for $A / k T=$ and $\lambda / r_{1}=0.1$; realistic system; $D_{0}=0.222, E=5.7 \times 10^{-5}: \square$, $i_{\max }=27, \phi=0.006 ; \triangle, 125,0.004 ;+, 512,0.006$.

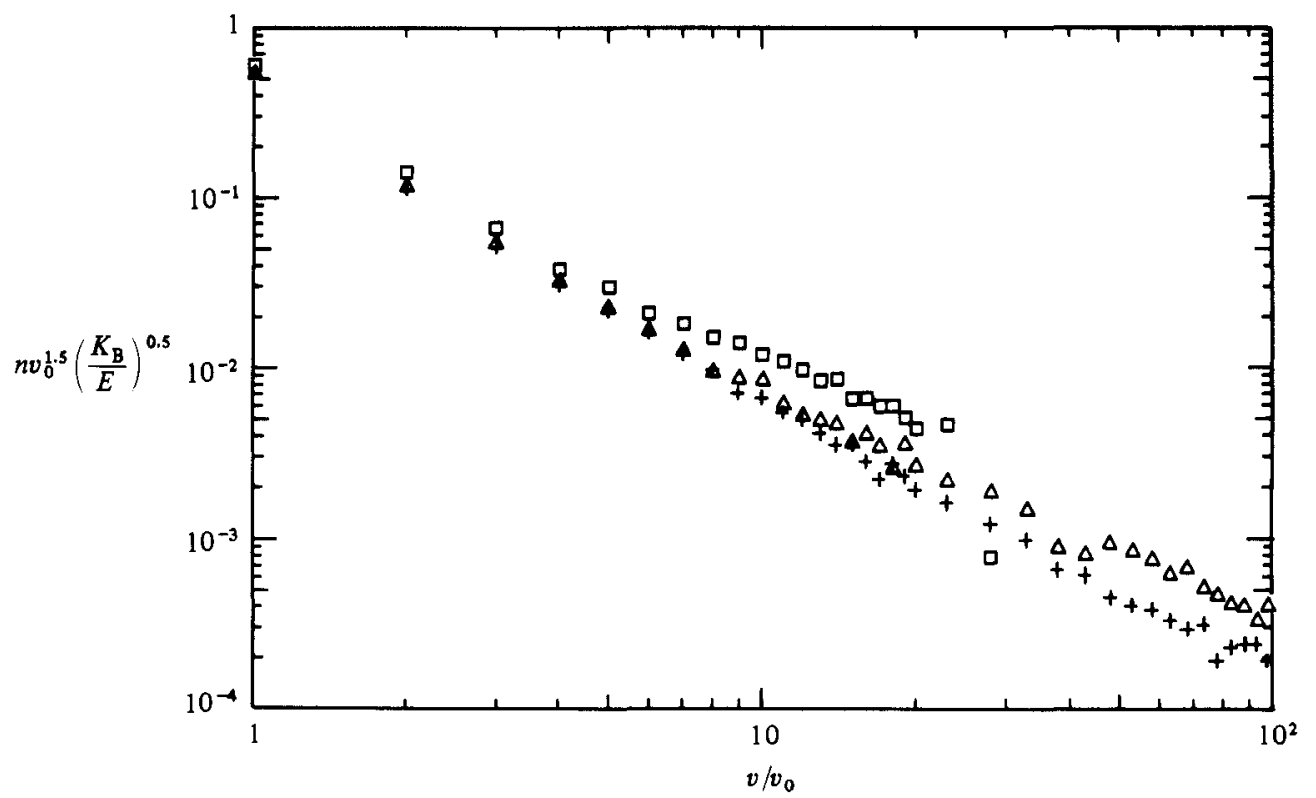

FigURE 4. Comparison of the steady-state non-dimensional size distribution for Brownian motion for different $i_{\max }$ for $A / k T=0.01$ and $\lambda / r_{1}=0.1$; realistic system; $D_{0}=0.222, E=5.7 \times 10^{-5}: \square$, $i_{\max }=27, \phi=0.006 ; \triangle, 125,0.005 ;+, 512,0.006$.

the best-fit line of slope $-\frac{3}{2}$ with the axis $v / v_{0}$ is considerably above the simulation runs of Part 1. This is shown in figure 5, where the results of two computer simulations at different $A / k T$ are compared with the non-interacting system of Part 1, all other parameters being the same.

At the upper end of the size range the results of all three simulation runs in both 


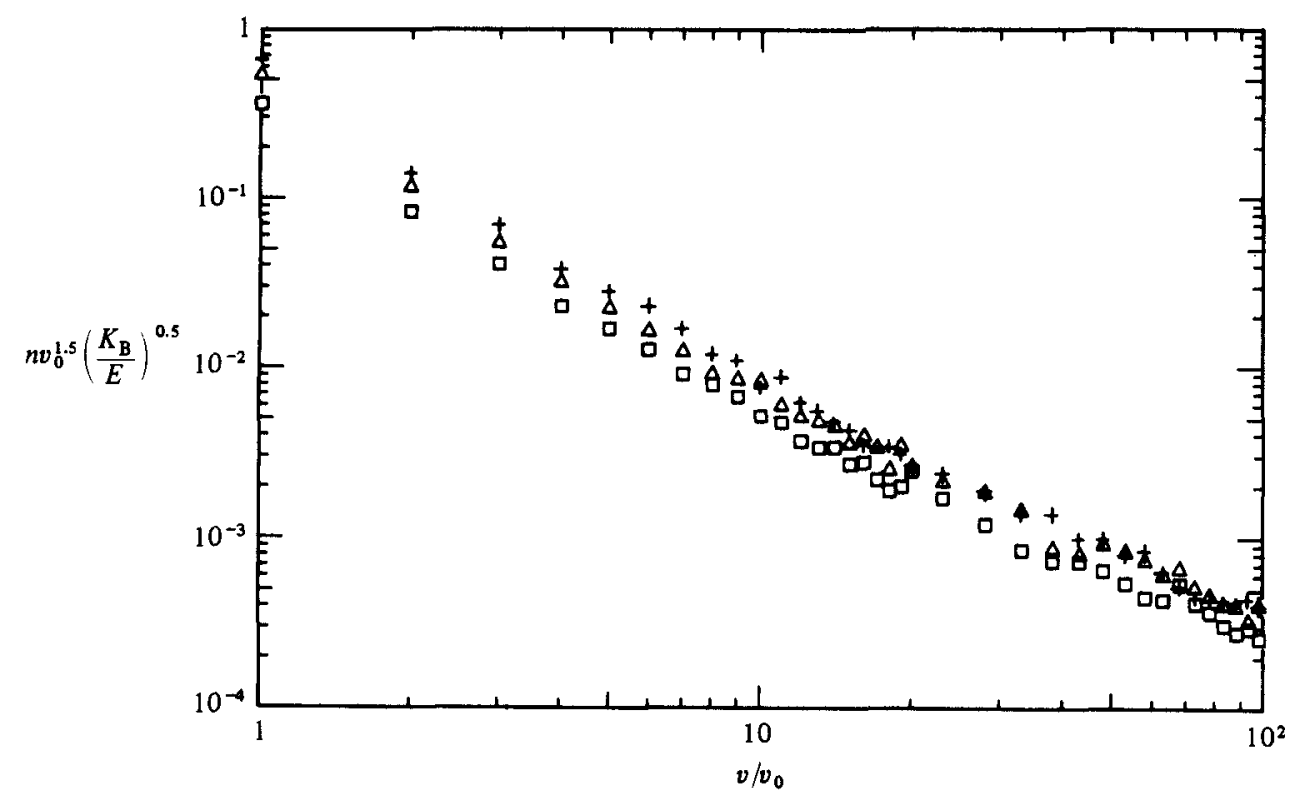

Figure 5. Comparison of the steady-state non-dimensional size distribution for a non-interacting system and for two realistic suspensions with different values of $A / k T ; D_{0}=0.222, E=5.7 \times 10^{-5}$, $i_{\max }=125: \square$, non-interacting system, $\phi=0.003 ; \triangle$, realistic system with $A / k T=1, \lambda / r_{1}=0.1$, $\phi=0.004 ;+$, realistic system with $A / k T=0.01, \lambda / r_{1}=0.1, \phi=0.005$.

figures 3 and 4 are statistically identical. It seems that the constant addition of unit particles, which clearly cannot represent properly the creation of unit particles by coagulation of smaller ones, covers the influence of $v_{\max }$ on the smallest particles of the simulation. For the largest part of the size range a constant decline in level of the size distribution with increasing $v_{\max } / v_{0}$ occurs in both figures 3 and 4 . Contrary to the 'non-interacting' particle system of Part 1 , the size range influences the final steady-state size distribution.

\section{Laminar shear}

Adler (1981) used the rigorous theory for the hydrodynamic interaction of two unequal spheres in simple shear flow (Batchelor \& Green 1972; Arp \& Mason 1976) to correct Smoluchowski's (1917) expression for the collision rate of spherical particles with radii $r_{1}$ and $r_{2}$ and number concentrations $N_{1}$ and $N_{2}$ per unit volume of fluid. The collision rate is

$$
{ }_{3}^{4} N_{1} N_{2}\left(r_{1}+r_{2}\right)^{3} G E_{\mathrm{sh}}\left(r_{1}, r_{2}\right)
$$

where $E_{\mathrm{sh}}\left(r_{1}, r_{2}\right)$ is Adler's (1981) correction factor (or collision efficiency). In Smoluchowski's rectilinear approach, geometrical exclusion determines the collision cross-section of the two particles. Hydrodynamic forces induce curvature in the particle trajectories which can be open or closed. Between the two kinds of trajectories a separation surface exists whose cross-section at infinite interparticle distance defines a 'curvilinear' collision cross-section. In the absence of other forces the cross-section of the separation surface tends to zero at large distances (Batchelor \& Green 1972), reflecting the singular behaviour of the interparticle hydrodynamic force in Stokes flow at particle contact. When, in addition, van der Waals' or other 


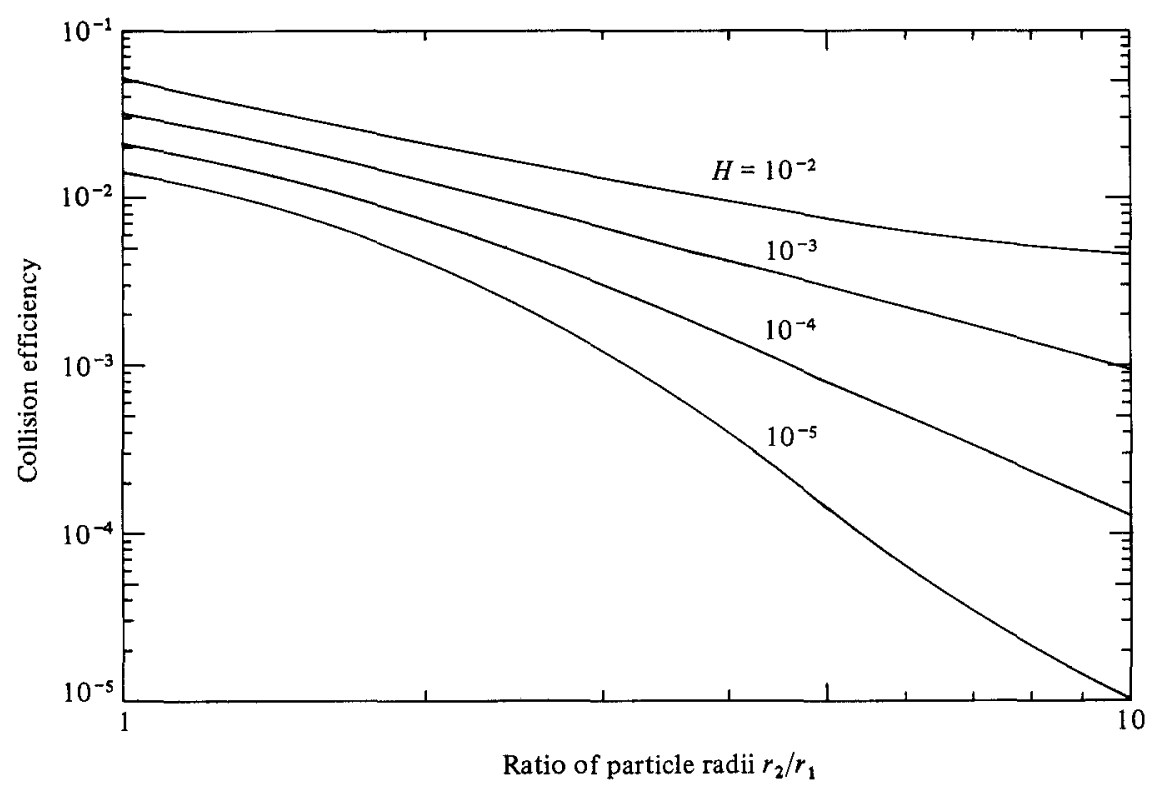

FIGURE 6. Collision efficiencies of spherical particles in simple shear (Adler 1981).

external forces act between the particles, a non-zero curvilinear cross-section may exist.

The correction $E_{\mathrm{sh}}\left(r_{1}, r_{2}\right)$ to the rectilinear collision rate is equivalent to defining a curvilinear collision cross-section $a^{2}$ :

$$
a^{2}=E_{\mathrm{Sh}}^{2}\left(r_{1}, r_{2}\right)\left(r_{1}+r_{2}\right)^{2} .
$$

For two unequal spherical particles in simple shear flow in the presence of van der Waals' forces $E_{\mathrm{sh}}\left(r_{1}, r_{2}\right)$ is a function of the relative size of the interacting particles and the dimensionless parameter

$$
H=\frac{A}{144 \pi \mu r_{2}^{3} G},
$$

where $A$ is the Hamaker constant, $G$ the rate of strain and $r_{2}$ the radius of the large particle. $H$ represents the relative strength of the attractive van der Waals' forces and the shear. The collision efficiency $E_{\mathrm{sh}}\left(r_{1}, r_{2}\right)$ is plotted in figure 6 against the relative size of the interacting particles for various values of $H$. Adler (1981) reports corrections to the rectilinear collision rate for four different relative particle sizes $r_{2} / r_{1}=1,2,5,10$ and for $H$ ranging from $10^{-2}$ to $10^{-5}$. Interpolation was used to obtain the collision rate corrections for intermediate values of $r_{2} / r_{1}$. Figure 6 indicates that homocoagulation (coagulation between particles of similar size) is favoured over heterocoagulation. The first requirement for the existence of a quasi-stationary size distribution in a coagulating system of particles is thus fulfilled.

The computer simulation model of Part 1 is used to study the evolution of the size distribution of a coagulating population of particles subjected to laminar shear and accounting for van der Waals' forces. It is assumed that the suspensions is destabilized so that double-layer forces are negligible. The correction to the curvilinear collision cross-section obtained from (4.2) is used in the simulation to check for particle collisions. 


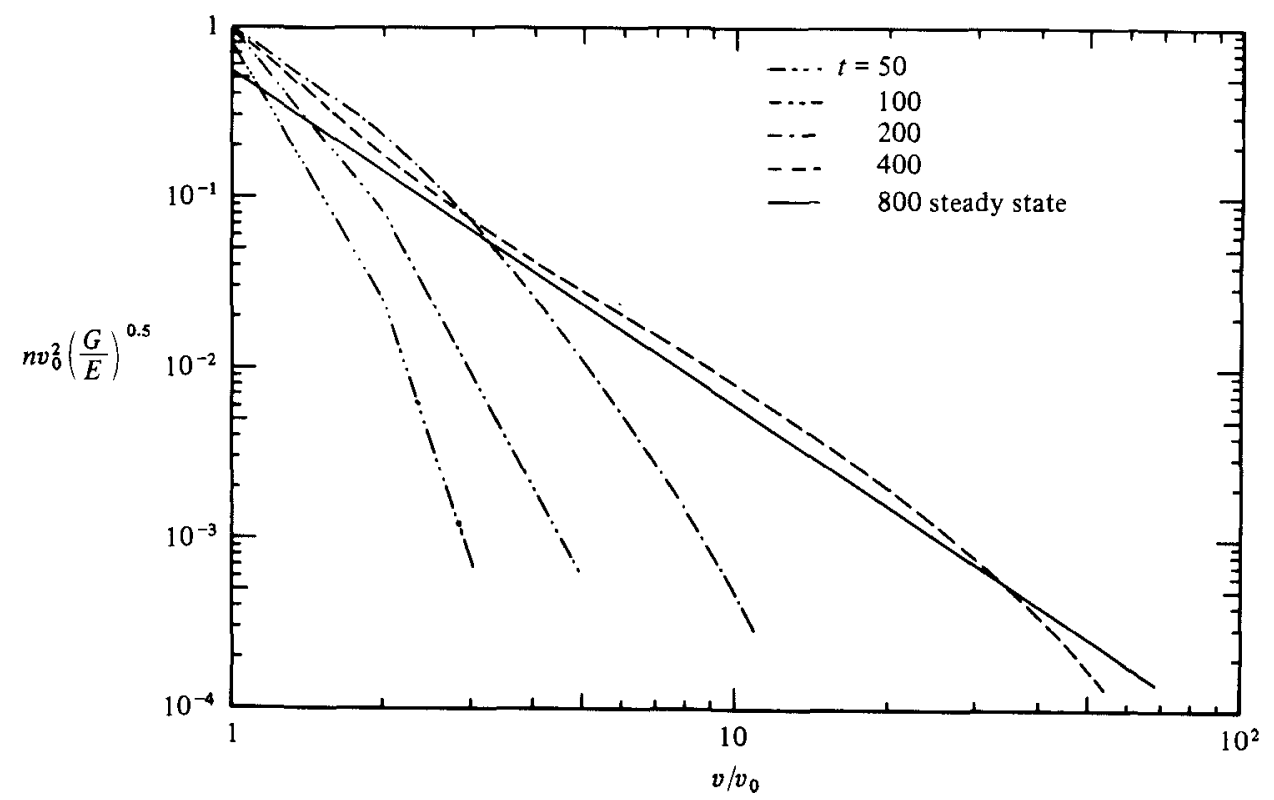

Fiaure 7. Evolution of the normalized size distribution for laminar shear; $H=10^{-2}, G=1$, $E=7.1 \times 10^{-5}, i_{\max }=125, N_{\text {initial }}=400$.

Figure 7 illustrates the evolution in time of an initially monodisperse suspension of particles with an initial volume concentration of $0.57 \%$ colliding under the influence of simple shear. The data of six simulation runs with identical initial conditions are averaged and normalized according to the dimensional arguments, (1.3), to give the plotted curves. The temporal development of the size distribution follows a pattern similar to the Brownian system; that is, the upper portion of the size spectrum attains a slope of -2 once a range of about one decade in volume is reached. Notice that the size distribution approaches its steady-state value long before a dynamic equilibrium is attained. If $v_{0}$ represents an aerosol particle with radius $1 \mu \mathrm{m}$, then the strain rate used corresponds to $G=125 \mathrm{~s}^{-1}$, and 125 time steps correspond to $1 \mathrm{~s}$ of real time; if $v_{0}$ is set equivalent to a micron-sized hydrosol particle, $G=2 \mathrm{~s}^{-1}$, and 2 time steps correspond to $0.1 \mathrm{~s}$ of real time. The difference in the physical interpretation of the results of the simulation in aerosol and aqueous systems is due to the dependence of the dimensionless parameter $H$ on viscosity.

Figure 8 is a comparison of the steady-state size distribution of three coagulating populations of particles when the maximum size $v_{\max }$, of a particle allowed to stay in the 'control' volume varies. For the three sets of data shown $H=10^{-2}$ and $v_{\max } / v_{0}=27,125$ and 512. The numerical results, non-dimensionalized according to (1.3) and averaged over 2000 time steps, collapse onto a slope of -2 . The three populations of particles are statistically identical: the size range does not influence the final steady-state size distribution.

The effect of the hydrodynamic interactions in decreasing the coagulation rate is illustrated in figure 9. The final steady-state size distribution of two populations of particles at $H=10^{-2}$ and $10^{-4}$ are compared with the non-interacting system of Part 1. The size distribution shifts upwards as the strength of the shear (i.e. rate of strain) decreases. Hydrodynamic and van der Waals forces merely change the value of the 'constant' $a_{\mathrm{sh}}$ in (1.3). According to Zeichner \& Schowalter (1977), $E_{\mathrm{sh}}\left(r_{1}, r_{2}\right)$ is 


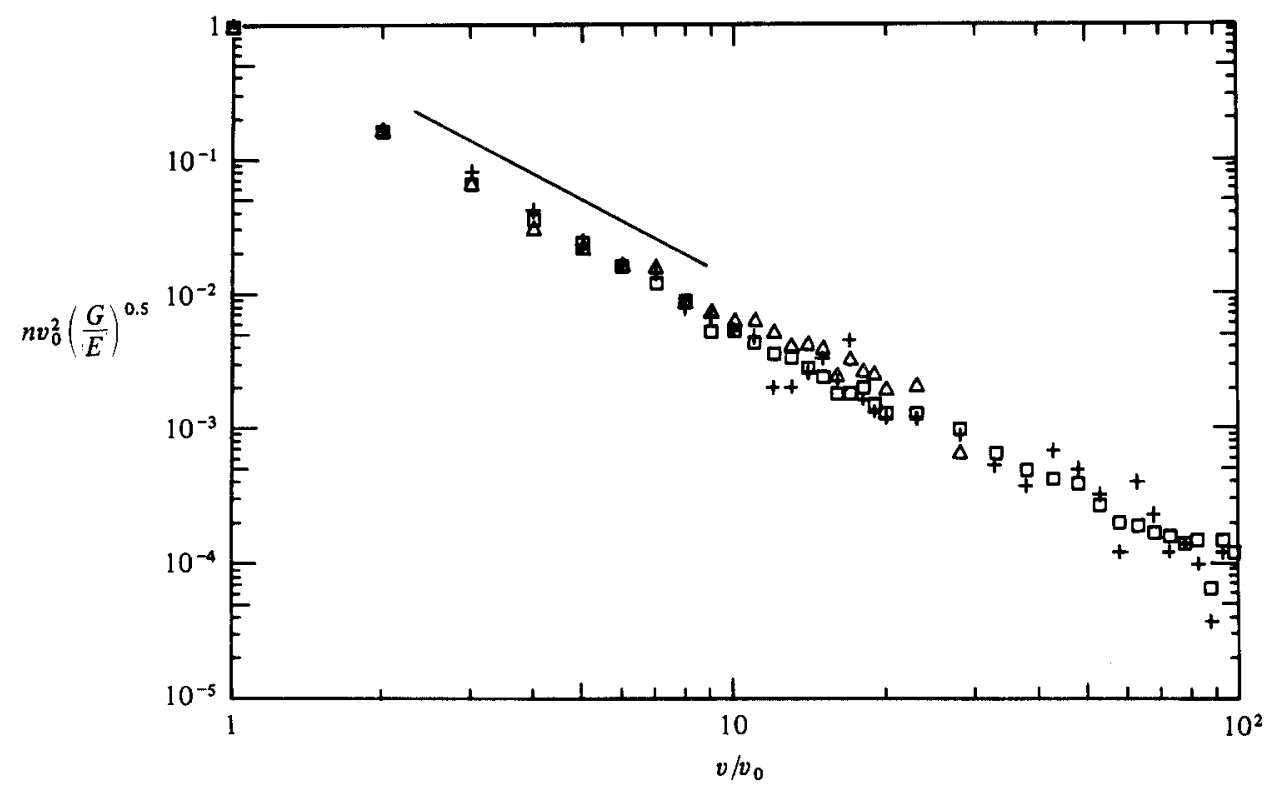

Figure 8. Comparison of the steady-state normalized size distribution for laminar shear for different values of $i_{\max } ; H=10^{-2}, G=1, E=7.1 \times 10^{-5} ; \triangle, i_{\max }=27, \phi=0.009 ;+, 125,0.010$; $\square, 512,0.011$.

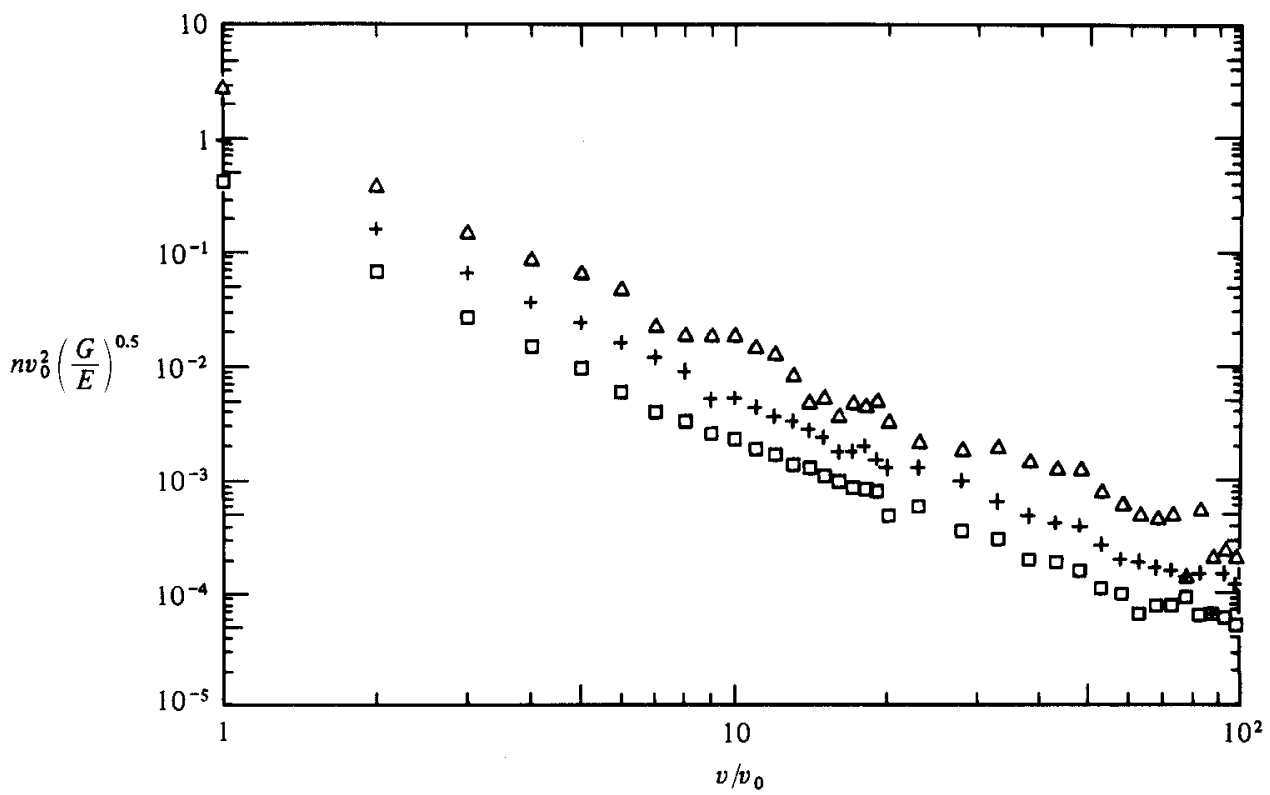

Figure 9. Comparison of the steady-state normalized distribution for laminar shear for a non-interacting system and for two realistic systems with different values of $H=A / 144 \pi \mu r^{3} G: \square$, non-interacting system, $G=1, E=1.1 \times 10^{-3}, i_{\max }=125, \phi=0.037 ; \quad+$, realistic system, $H=10^{-2}, G=1, E=7.1 \times 10^{-5}, i_{\max }=125, \phi=0.010 ; \triangle$, realistic system, $H=10^{-4}, G=1$, $E=1.4 \times 10^{-5}, i_{\max }=125, \phi=0.027$. 
proportional to $H^{\alpha}$, where $\alpha=0.23$, and therefore the collision function $\beta$ scales as $G^{-\frac{1}{2}(1-\alpha)}$, and hence $n(v)$ is proportional to $v^{-2+\frac{1}{2} \alpha}$. Hunt's (1982) dimensional arguments are then modified, and from (1.8) we obtain

$$
n(v)=a_{\mathrm{sh}}^{\prime} \frac{E^{\frac{1}{2}}}{G^{1}\left(1-\alpha A^{\prime}\right.} v^{-2+\frac{1}{2} \alpha}\left(\frac{144 \pi \mu}{A}\right)^{\frac{1}{2} \alpha}
$$

for laminar-shear-induced coagulation when hydrodynamic and van der Waals' interactions are taken into consideration. The change in the slope of the steady-state size distribution suggested by (4.4) is very small to be detected by the Monte Carlo simulation. Nevertheless, the values of the parameters $r_{0}, G, E$ and $H$ used in the simulation appearing in figure 8 yield for the constant $a_{\mathrm{sh}}^{\prime}$

and

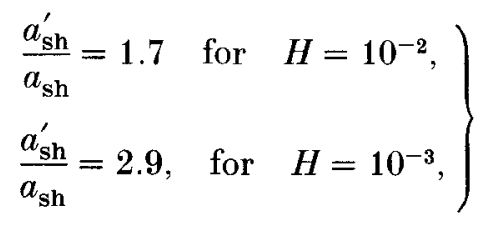

which hold approximately for the constants $a_{\mathrm{sh}}^{\prime}$ and $a_{\mathrm{sh}}$ obtained from the results of the simulations.

\section{Differential sedimentation}

\subsection{Hydrodynamic interactions and computer simulation}

In contrast with Brownian diffusion and fluid shearing, differential sedimentation induced coagulation involves a physical property of the particles : their density-excess ratio $\left(\rho_{\mathrm{p}}-\rho_{\mathrm{f}}\right) / \rho_{\mathrm{f}}$ over that of the fluid. Collisions and subsequent coagulation may occur when larger or heavier particles overtake smaller ones.

The presence of a particle moving with velocity $u$ induces a velocity gradient of order $u r / s^{2}$ at a distance of $s$ in the surrounding fluid (Batchelor 1976). This velocity gradient modifies the trajectory of an approaching particle as if a force dipole were located at the position of the particle. The collision rate, per unit volume of fluid, of particles with sizes $r_{1}$ and $r_{2}$ is given by the rectilinear collision function for differential settling (table 1) multiplied by the number densities $N_{1}$ and $N_{2}$ of the particles and the collision efficiency $E_{\mathrm{ds}}\left(r_{1}, r_{2}\right)$ :

$$
\text { collision rate }=\frac{2}{9} \pi k_{\mathrm{ds}}\left(r_{1}+r_{2}\right)^{2}\left|\left(r_{1}^{2}-r_{2}^{2}\right)\right| N_{1} N_{2} E_{\mathrm{ds}}\left(r_{1}, r_{2}\right) .
$$

Published work dealing with hydrodynamic interactions of settling particles is in connection with gravitational coagulation of water droplets in air. Theoretical computations of the collision efficiency are based on several schemes, such as assuming Stokes flow, using Oseen's equations or using the slip-corrected Stokes flow equations (in order to account for the breakdown of continuum flow when the separation of the particles becomes of the order of the mean free path) giving, interestingly, no markedly different results (see Pruppacher \& Klett 1978), unless the particles are of nearly equal size. Experimental difficulties have not allowed verification of the computed-collision efficiencies in the laboratory, mainly because of the critical role which molecular or other short-range forces play in coalescing two particles which are brought into contact by their relative motion (Tag 1976). Neiburger et al. (1976) obtained an analytic expression for theoretical collision efficiencies, computed assuming Stokes flow with the slip-flow correction for small particles $\left(r_{2} \leqslant 30 \mu \mathrm{m}\right)$ 
and approximating the flow fields of larger particles by a superposition technique in order to include the effects of inertia:

$$
E_{\mathrm{ds}}=E_{0}+E_{1}+E_{2}+E_{4}
$$

where

$$
\begin{aligned}
& E_{0}=0.95-\left(0.7-0.005 r_{2}\right)^{4}\left(7.92-0.12 r_{2}+0.001 r_{2}^{2}\right), \\
& E_{1}=-\left(\frac{r_{1}}{r_{2}}-0.5\right)^{2} \\
& E_{2}=-1.5 \exp \left[-\left(0.0015 r_{2}^{2}+8\right) \frac{r_{1}}{r_{2}}\right] \\
& E_{3}=-\left(1-0.007 r_{2}\right) \exp \left[-0.651 r_{2}\left(1-\frac{r_{1}}{r_{2}}\right)\right] \\
& E_{4}= \begin{cases}0 & \left(r_{2}<20 \mu \mathrm{m}\right), \\
\exp \left[-30\left(1-\frac{r_{1}}{r_{2}}\right)\right] \quad\left(r_{2} \geqslant 20 \mu \mathrm{m}\right)\end{cases}
\end{aligned}
$$

$E_{\mathrm{ds}}$ is plotted in figure 10 as a function of the particle ratio $p=r_{1} / r_{2}\left(r_{2}>r_{1}\right)$ for different $r_{1}$. For fixed relative particle size the collision efficiency $E_{\mathrm{ds}}$ increases with increasing particle size since the deflecting hydrodynamic forces become less important as particle inertia increases. For the same reason $E_{\mathrm{ds}}$ decreases with $p$ when $p \ll 1$, for fixed $r_{2}$. For $p$ nearer unity 'wake' capture occurs when the two particles are large enough for inertial effects to become appreciable.

The collision efficiencies given by (5.2) are reasonably accurate for water droplets in air, but their applicability to other particle systems is questionable. For example, double-layer forces are ignored, and inertial effects are more important for water droplets in air than for solid particles in water. The slip-flow theory involves the assumption that the tangential fluid velocity at the surface of the particle is smaller than the surface velocity of the particle. This velocity 'slip' is related to the local stress and the mean free path of the fluid, the factor of proportionality depending largely on the properties of the particle surface. Flocculated particles in water may have a non-spherical shape and are often porous. Thus, although the collision efficiencies in (5.2) may have the qualitatively correct dependence on the absolute and relative size of the interacting particles, they should be used with caution for particle suspensions other than water droplets in air.

The coagulation process was simulated by imposing on each spherical particle its Stokes terminal settling velocity

$$
w=\frac{2}{9} \frac{g\left(\rho_{\mathrm{p}}-\rho_{\mathrm{f}}\right)}{\mu} r^{2}
$$

valid for timescales greater than the particle viscous relaxation time $t_{\mathrm{r}}=\frac{2}{9} r^{2} / \nu$. All particles have the same density and are moving in a 'control volume' of variable dimensions. Particles reaching the bottom are reintroduced at the top at a random cross-sectional position. This is necessary in order to prevent the simulation from becoming deterministic after a certain time: collisions would cease after each particle had swept out its own path through the control volume. Particles move in straight paths during the time step $\Delta t$. Equation (5.1) suggests that hydrodynamic interactions can be incorporated in the simulation by using an effective collision cross-section

$$
E_{\mathrm{ds}}\left(r_{1}, r_{2}\right)\left(r_{1}+r_{2}\right)^{2}
$$




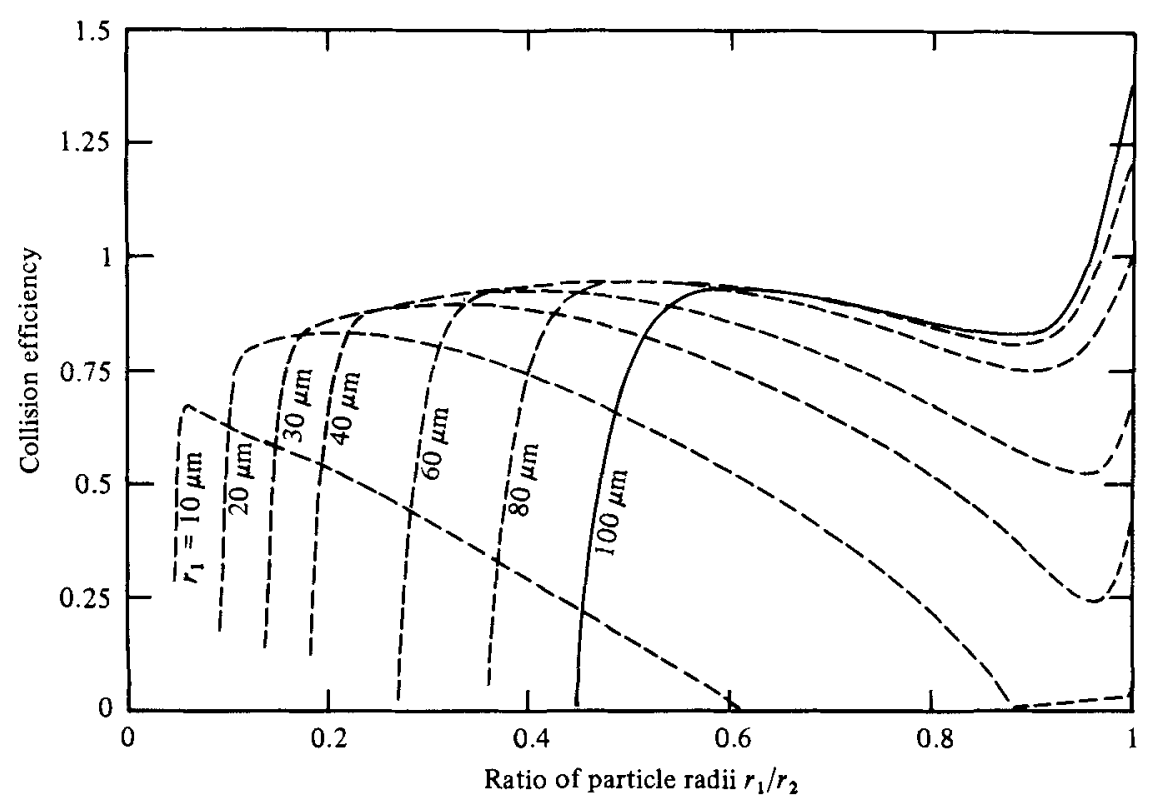

FigURe 10. Collision efficiencies of sedimenting particles of various sizes (Neiburger et al. 1976). In contrast with the method used commonly in cloud physics literature, the collision efficiencies are plotted for specific values of $r_{1}$ rather than $r_{2}$, since this facilitates their use in the simulations, where $r_{1}$ corresponds to the unit particle.

to check for particle collisions. Notice, however, that this formulation assumes that collisions between particles of equal size do not occur even when their collision efficiency is non-zero, thus ignoring wake capture.

The algorithm was verified using a non-coagulating version of the simulation with two particle sizes. The collision rates computed from the simulation were in agreement with the prediction of the theoretical model (see figure 18 in $\$ 6$ ).

An initially monodisperse system of spherical particles was subjected to gravity settling. Weak Brownian diffusion or weak fluid shearing operated at the same time to initiate the coagulation process. When uniform shearing motion $u=G x$ is imposed in the presence of settling, the particle crosses the streamlines perpendicular to the direction of the shearing during the time step $\Delta t$. The particle displacement $\boldsymbol{Y}(i)$ in any time step is then

$$
\left.\begin{array}{c}
Y(\mathrm{i})-\left(Y_{1}(i), 0, Y_{3}(i)\right), \\
Y_{1}(i)=G\left(P_{3}(i)+0.5 Y_{3}(i)\right) \Delta t, \quad Y_{3}(i)=\frac{2}{9} K_{\mathrm{ds}} r^{2} \Delta t,
\end{array}\right\}
$$

where $\boldsymbol{P}(i)=\left(P_{1}(i), P_{2}(i), P_{3}(i)\right)$ is the position of the particle $i$ at the beginning of the time step. It is necessary to take into account the 'average' vertical position of the particle during any time step $\Delta t$ to predict the collision rates correctly.

\subsection{Simulation results}

Figure 11 shows the steady-state size distributions of two initially monodisperse systems subjected to weak Brownian motion and weak laminar shearing respectively, and to gravity settling (by 'weak' we mean that the collision rate due to 'weak' Brownian motion or 'weak' laminar shear is smaller than the collision rate due to differential sedimentation for particles $r_{1}$ and $r_{2}$ when $r_{2} / r_{1} \geqslant 2$ ). Hydrodynamic 


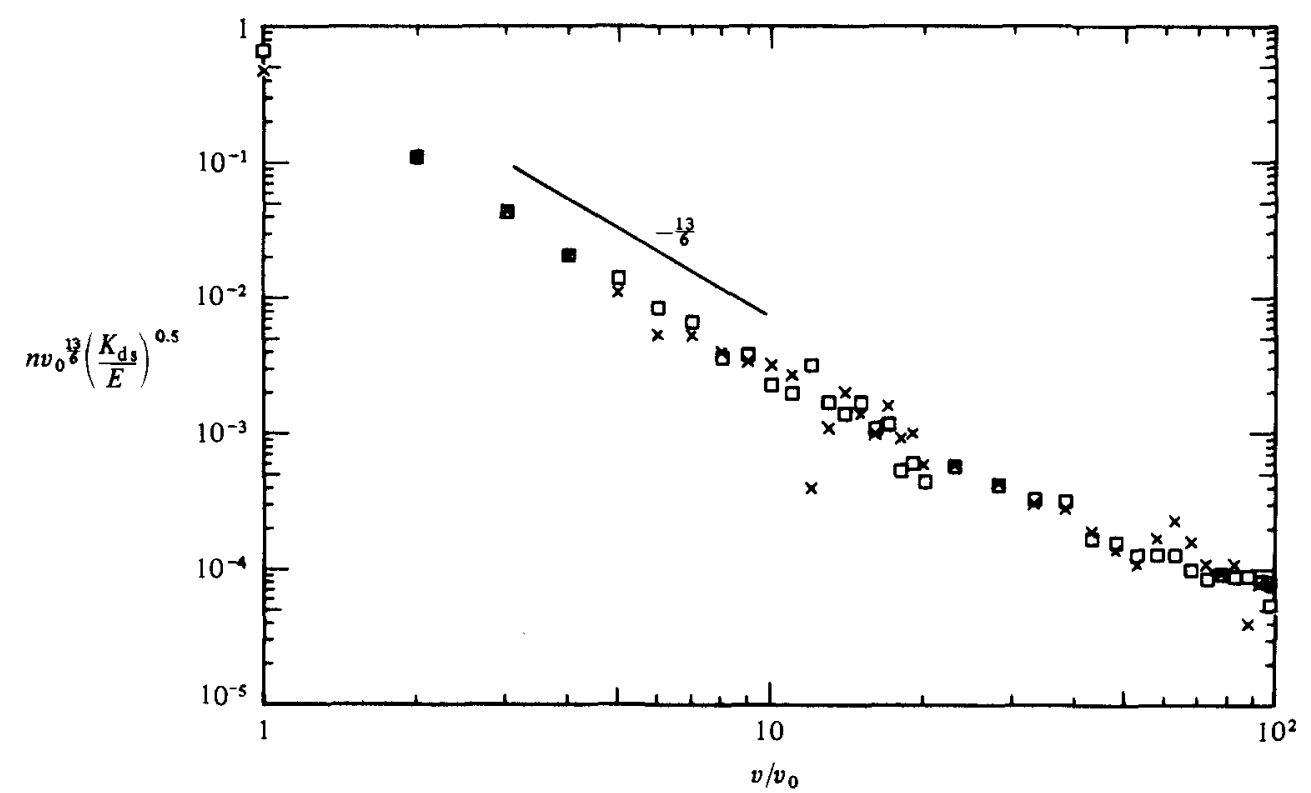

Figure 11. Non-dimensional steady-state particle size distributions for differential sedimentation and weak Brownian motion or weak laminar shear; non-interacting suspensions: $\square, K_{\mathrm{ds}}=0.94$, $G=2, E=5.6 \times 10^{-5}, \quad i_{\max }=125, \phi=0.005 ; \quad+, \quad K_{\mathrm{ds}}=0.94, \quad D_{0}=0.222, \quad E=1.4 \times 10^{-5}$, $i_{\max }=125, \phi=0.005$.

interactions such as discussed in $\$ \S 3$ and 4 are initially ignored but will be discussed later. The size distributions are collapsed when non-dimensionalized according to (1.4) and plotted against particle volume, non-dimensionalized with the unit particle volume. A constant $-\frac{13}{6}$ slope line is drawn for comparison. The data shown in figure 11 are results of the simulation averaged over 1600 time steps. A long-time average is needed to reduce the scattering of the data at the long tail of the distribution caused by the high collision probability of the large particles.

The next figure illustrates how weak Brownian motion modifies the size distribution at the small size range. The steady-state size distribution of the population of particles subjected to weak Brownian motion and gravity settling (figure 11) is allowed to evolve in the presence of settling only. The steady-state size distribution attained and averaged over 100 time steps is compared with the initial one in figure 12. The numerical results are statistically identical in the largest part of the size spectrum. When only differential settling operates as a volume-transferring mechanism through the size spectrum, the shape of the size distribution near the small-size range reflects the ineffectiveness of differential settling to coagulate particles of similar size. Particles of equal size subjected to gravity settling do not collide. However, since the flux of particle volume into the size range from coagulation of particles smaller than $v_{0}$ is represented in the simulation by a constant addition of unit particles, it is apparent that this scheme cannot represent properly the collisions of particles larger than $v_{0}$ with particles smaller than $v_{0}$; hence the awkwardly high number of unit particles in the size distribution shown in figure 12 .

Figures 13 and 14 show two states in the development of the size distribution of an initially monodisperse system of particles undergoing Brownian diffusion and settling. The relative strength of the two coagulation mechanisms can be assessed 


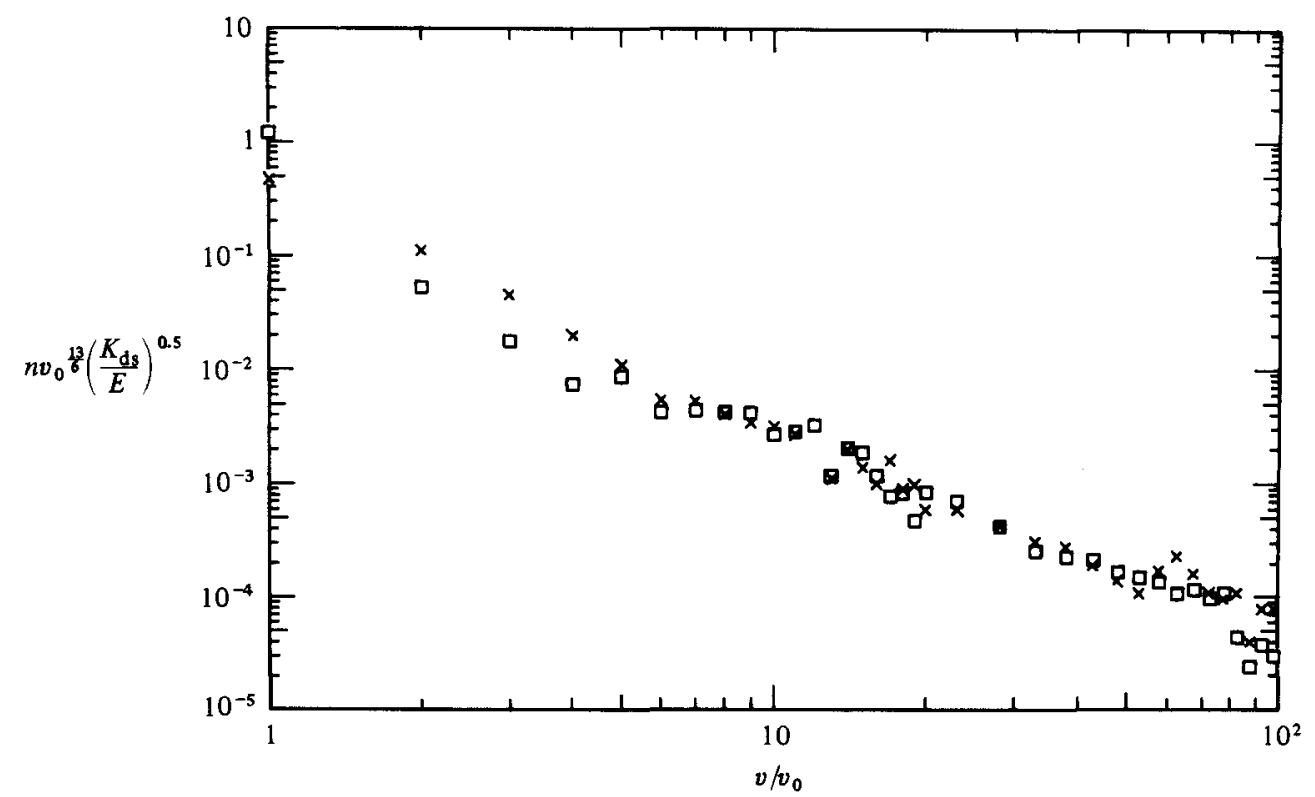

Figure 12. Comparison of the steady-state normalized size distribution for differential settling and Brownian motion with differential settling; $K_{\mathrm{ds}}=0.94, E=5.6 \times 10^{-5}, i_{\max }=125 ; \square, D_{0}=0$, $\phi=0.006 ;+, D_{0}=0.222, \phi=0.005$.

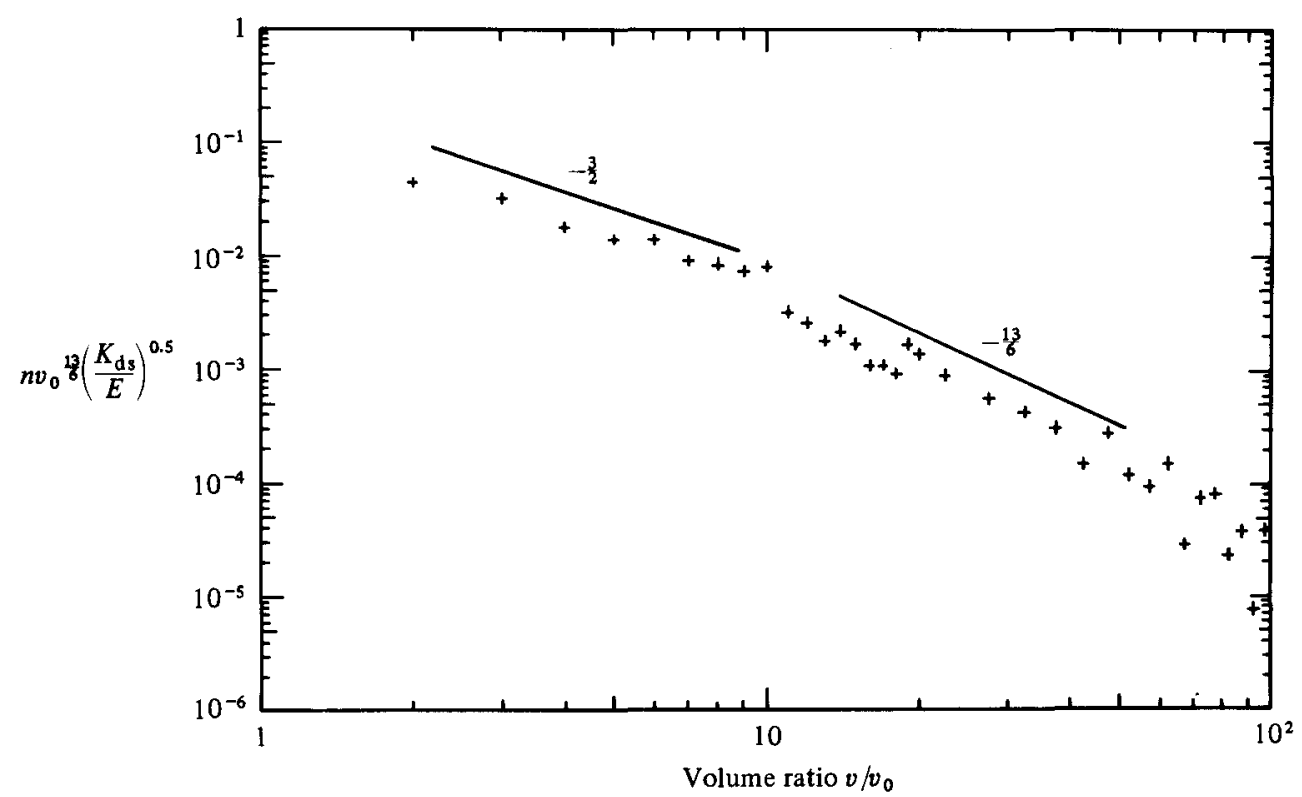

FraURE 13. Non-dimensional size distribution for differential sedimentation and Brownian motion at 1200 time steps; $K_{\mathrm{ds}}=0.19, D_{0}=0.222, N_{\text {initial }}=200, E=5.6 \times 10^{-5}, i_{\max }=125$.

from the ratio of their respective rectilinear collision functions $\beta_{\mathrm{B}}$ and $\beta_{\mathrm{ds}}$ (see table 1)

$$
\frac{\beta_{\mathrm{B}}}{\beta_{\mathrm{ds}}}=18 \frac{D_{0}}{K_{\mathrm{ds}}} \frac{1}{r_{0}^{3}} \frac{1}{q\left(q^{2}-1\right)},
$$

where $q$ is the particle radius non-dimensionalized with the radius $r_{0}$ of the unit particle, and $D_{0}$ is the diffusivity of the unit particle. The transition in dominance 


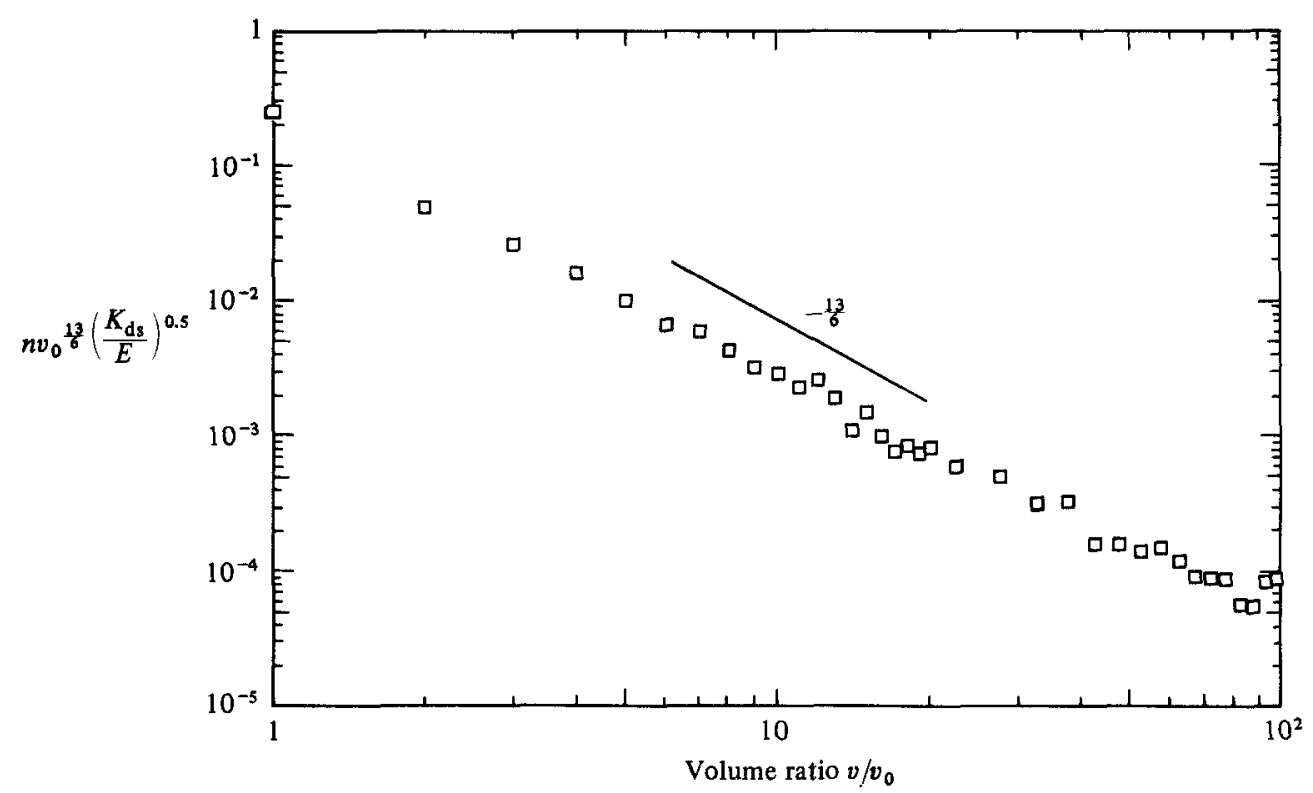

Figure 14. Non-dimensional steady-state size distribution for differential sedimentation and Brownian motion; $K_{\mathrm{ds}}=0.19, D_{0}=0.222, N_{\text {initial }}=200, E=5.6 \times 10^{-5}, i_{\max }=125, \phi=0.016$.

of the two mechanisms in the particle system shown in figures 13 and 14 is at $v / v_{0}=24$ : the collision rates of particles of volume $24 v_{0}$ with particles of volume $v_{0}$ due to Brownian motion and differential settling are equal. Figure 13 shows the particle size distribution after 1200 time steps, only about 200 time steps before a steady state is attained. The $-\frac{3}{2}$ and $-\frac{13}{6}$ slopes are clearly distinguishable, but the transition point is shifted from $v / v_{0}=24$, indicating that the influence of the large particles undergoing differential settling induced coagulation tends to propagate to smaller size ranges in the size spectrum. The statistically steady state attained is shown in figure 14. The data points shown are averaged over 3000 time steps after the first large particle was lost from the 'control' volume. The dominance of differential settling is evident.

So far hydrodynamic interactions have been ignored. We turn now to more realistic particle systems in which hydrodynamic forces between two approaching particles exist. The time evolution of the normalized size distribution of an initially monodisperse suspension subjected to gravity settling and weak Brownian diffusion is shown in figure 15. The data of five simulation runs, for a value of $r_{0}$ corresponding to an actual particle radius of $40 \mu \mathrm{m}$, are averaged and smoothed to give the curves shown. For a unit particle with radius $40 \mu \mathrm{m}$ and a density-excess ratio $\left(\rho_{\mathrm{p}}-\rho_{\mathrm{p}}\right) / \rho_{\mathrm{f}}=0.9,1 \mathrm{~s}$ of real time corresponds to about 376 time steps in the simulation (considering the dimensionless group $K_{\mathrm{ds}} t r_{0}$ ). The development pattern is strikingly similar to the Brownian and shear systems, but the change in the number of unit particles is more significant. This indicates that large particles formed at progressively later times influence significantly the particle-size distribution at the small end of the spectrum.

The functional dependence of the effective collision cross-section on $r_{1}$ (figure 10) suggests that the shape of the size distribution will depend on the absolute size of the particles. This is illustrated in figure 16 , where the normalized size distributions of two particle systems differing in the size of the unit particles are compared. The 


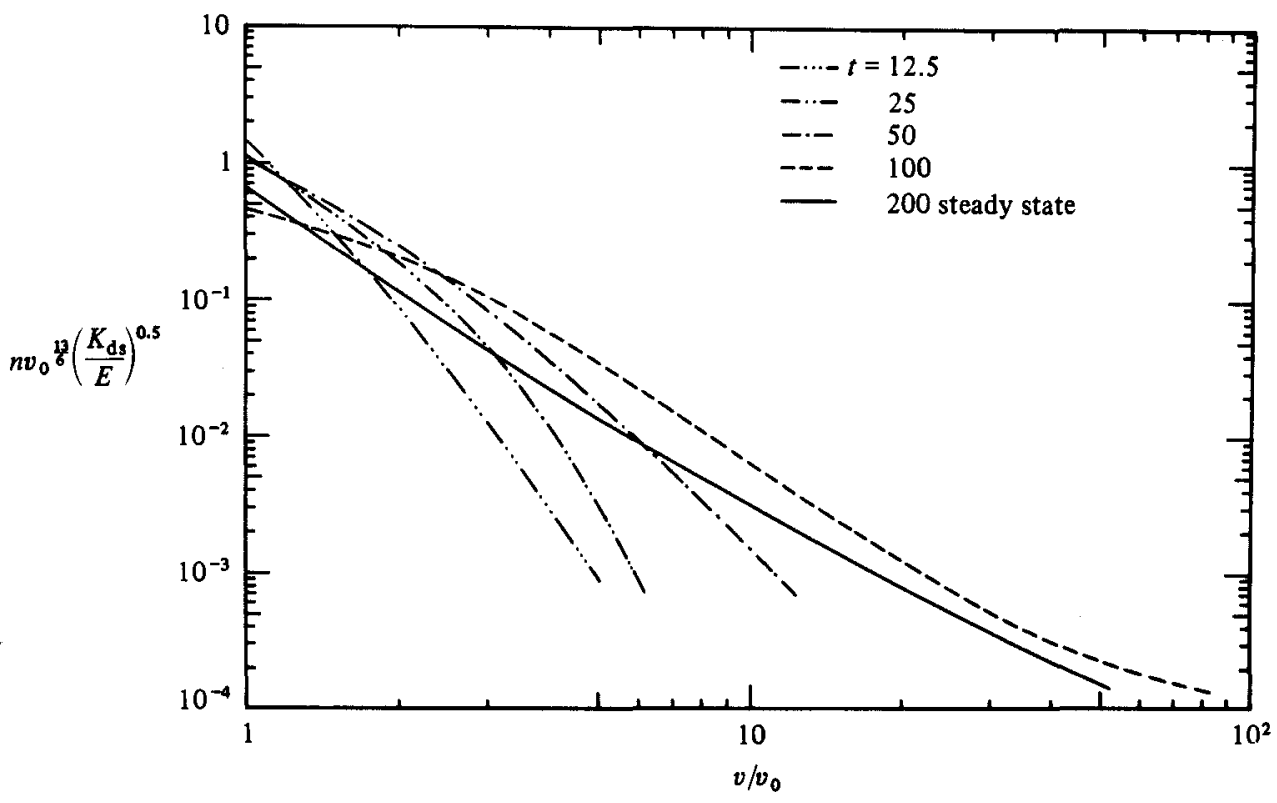

FigURE 15. Evolution of the normalized size distribution for differential sedimentation; $K_{\mathrm{ds}}=0.94, D_{\mathrm{0}}=0.222, E=5.6 \times 10^{-5}, i_{\max }=125$.

two sets of data correspond to actual unit particle sizes of 20 and $40 \mu \mathrm{m}$, all other parameters being equal. The plotted points are numerical data averaged over 1000 time steps and normalized as suggested by (1.4). Weak Brownian diffusion is allowed to operate in order to smooth the size distribution at the smaller particle-size range. The smaller the size of the unit particles, the steeper the final steady-state size distribution becomes. In figure 17 two 'interacting' populations of particles with $r_{0}=20$ and $80 \mu \mathrm{m}$ are compared with a 'non-interacting' system. Note that for the latter the absolute size of the particles is irrelevant. There is an accumulation of particles in the size distribution with $r_{0}=80 \mu \mathrm{m}$ near $v / v_{0}=20$, where the cutoff in the respective efficiency curve occurs (see figure 10). From figures 16 and 17 we conclude that the slope of the size distribution of a coagulating system of suspended particles subjected to differential settling depends on the size of the particles. When the radius of the smallest particles involved in the simulation is less than about $40 \mu \mathrm{m}$, the steady-state size distribution has a slope steeper than $-\frac{13}{6}$; in simulations with larger $r_{0}$ the size spectrum is flatter.

In simulations performed with $r_{0}$ less than $15 \mu \mathrm{m}$ a steady-state size distribution was not attained, unless weak Brownian motion operated. Irrespective of the shape of the initial particle spectrum, the number of unit particles in the control volume constantly increased when only differential sedimentation-induced collisions were allowed to occur. This is due to the shape of the efficiency curve for $r_{1}$ less than about $15 \mu \mathrm{m}$; collisions simply do not occur for particles close in size and widely different in size. However, for particles less than $15 \mu \mathrm{m}$ shearing motion is more effective in inducing collisions (Hunt 1982).

Simulations performed for a non-interacting system of particles gave

$$
a_{\mathrm{ds}}=0.45 \pm 0.02
$$

for the dimensionless constant $a_{\mathrm{ds}}$ in (1.4). Hydrodynamic interactions between the approaching particles steepen or flatten the steady-state size distribution, depending 


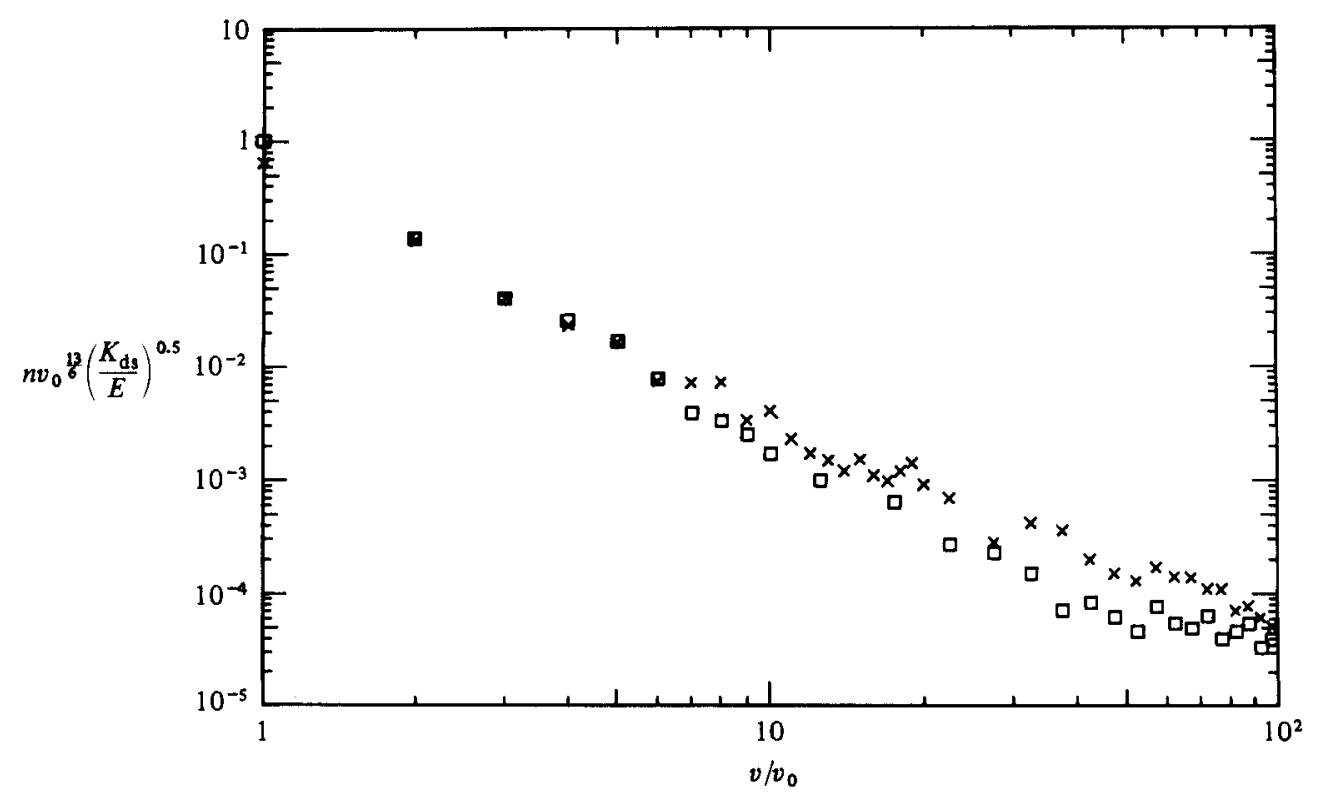

Figure 16. Comparison of the steady-state normalized size distribution for differential sedimentation and weak Brownian motion when different collision efficiencies are used; $K_{\mathrm{ds}}=0.94, D_{0}=0.222$, $E=5.6 \times 10^{-5}, i_{\max }=125: \square$, when $r_{0}$ corresponds to an actual radius of $20 \mu \mathrm{m}, \phi=0.004 ; \times$, when $r_{0}$ corresponds to an actual radius of $40 \mu \mathrm{m}, \phi=0.005$.

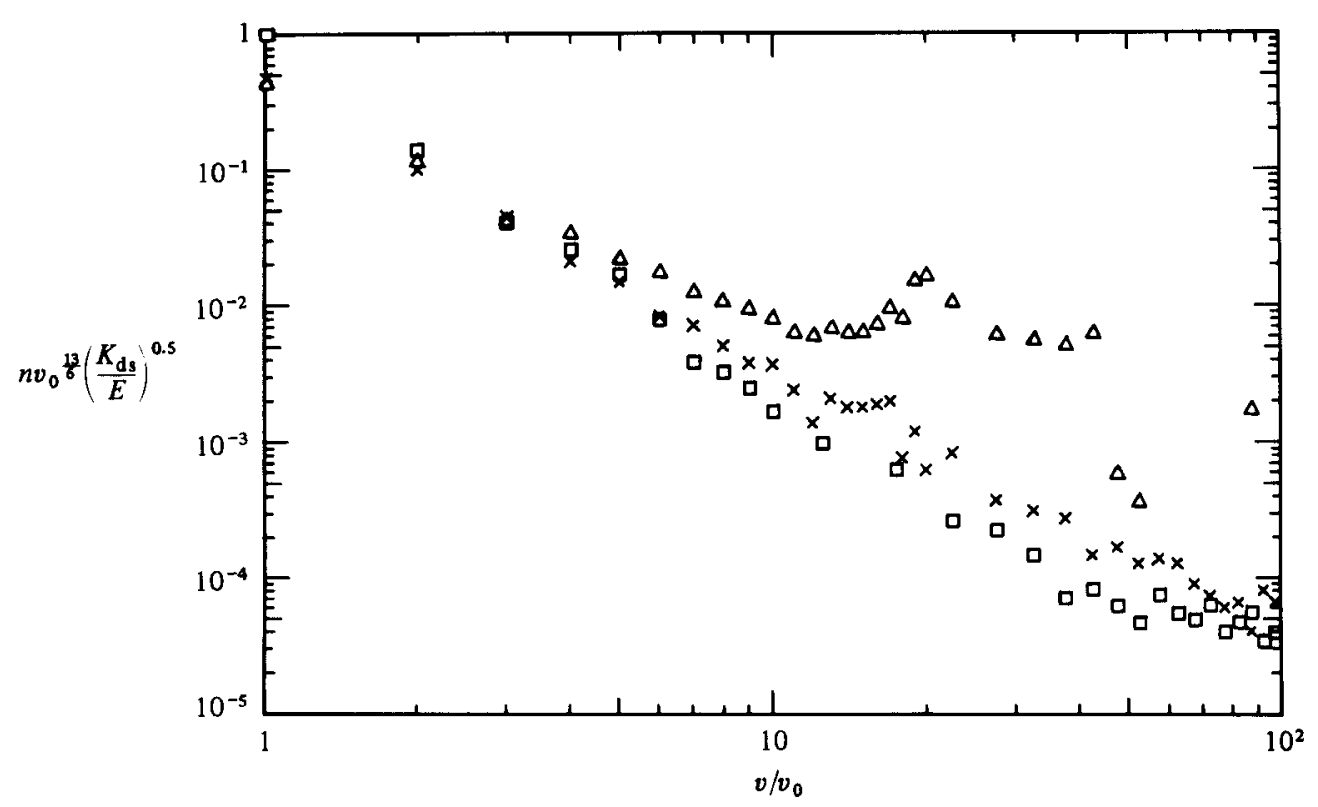

Figure 17. Comparison of the steady-state normalized size distribution for differential sedimentation and weak Brownian motion for a non-interacting system and two realistic ones; $K_{\mathrm{ds}}=0.94$, $D_{0}=0.222, E=5.6 \times 10^{-5}, i_{\max }=125: \times$, non-interacting, $\phi=0.010 ; \square$, realistic with $r_{0}$ corresponding to an actual radius of $20 \mu \mathrm{m}, \phi=0.004 ; \triangle$, realistic with $r_{0}$ corresponding to an actual radius of $80 \mu \mathrm{m}, \phi=0.005$. 
on the particle-size range considered. However, computational cost effectively prohibited the direct simulation of a more extended particle-size range. The simulations performed therefore involve overlapping sections of the size spectrum. The numerical results indicate that the size distribution becomes steeper the smaller the size of the particles considered; for unit particles smaller than about $15 \mu \mathrm{m}$ the computer model suggests that no steady state can exist as a result of the shape of the efficiency curve for such particles. Thus no power-law expression of the form of (1.4) with a unique exponent can represent the particle-size distribution in the size range where differential settling dominates. Unlike shear-induced coagulation (see $\S 4$ ), hydrodynamic interactions cannot be incorporated solely in the dimensionless coefficient $a_{\mathrm{ds}}$.

\section{Conclusions and discussion}

The direct simulation of the physical processes of particle collision and coalescence was undertaken in order to investigate Friedlander's (1960a,b) and Hunt's (1982) theory regarding the existence of a quasi-stationary particle-size distribution in aerosols and hydrosols. Observations in the atmosphere (Friedlander $1960 a, b)$ and in oceanic waters and wastewater sludges (Hunt 1982) and Hunt's experiments partly support the theory. The numerical simulations of Part 1 showed that, provided hydrodynamic and other interparticle forces are ignored, a population of coagulating particles can reach a state of dynamic equilibrium sustained by the flux of mass through the size space, when the collision mechanism is Brownian motion, simple shear or isotropic turbulent shear. The steady-state size distributions obtained in Part 1 were in agreement with Hunt's dimensional results.

This study re-examined the kinetics of a population of coagulating particles accounting for the influence of interparticle forces on the collision rate. Such forces can arise from the disturbance the presence of the particle causes in the fluid (hydrodynamic forces), from the cloud of ions that surround an electrically charged particle (double-layer forces), or they can be of molecular origin (van der Waals forces). These forces modify the trajectory of two approaching particles, increasing or decreasing the probability of collision and subsequent coalescence. The significance of these interactions for the validity of the theory lies in the functional dependence of the collision efficiency - which multiplies the rectilinear collision rate and incorporates the effect of all interparticle forces on the collision process - on the relative size of the interacting particles. For underlying Hunt's dimensional arguments is the notion that the coagulation process is mainly 'local' in size space.

The analytic estimates for the rectilinear collision function for Brownian diffusion, fluid shear and differential sedimentation induced coagulation were reproduced in this study using the non-coagulating version of the model. This is illustrated in figure 18, where the computed number of collisions, for several collision mechanisms, is plotted against the number of collisions predicted by the theoretical models. The data points shown are results from simulations involving a variety of different situations, such as monodisperse systems or suspensions with two particle sizes and systems with different densities and/or with different values of the dimensional parameters $K_{\mathbf{B}}$, $G$ and $K_{\mathrm{ds}}$ (which represent the strength of the collision mechanisms).

For Brownian-motion-induced coagulation, collision efficiencies were computed for two spherical particles of different size, assuming Stokes flow and taking into account the attractive van der Waals and the double-layer forces. The latter are assumed dispersive, since suspended particles in natural waters usually carry a negative 


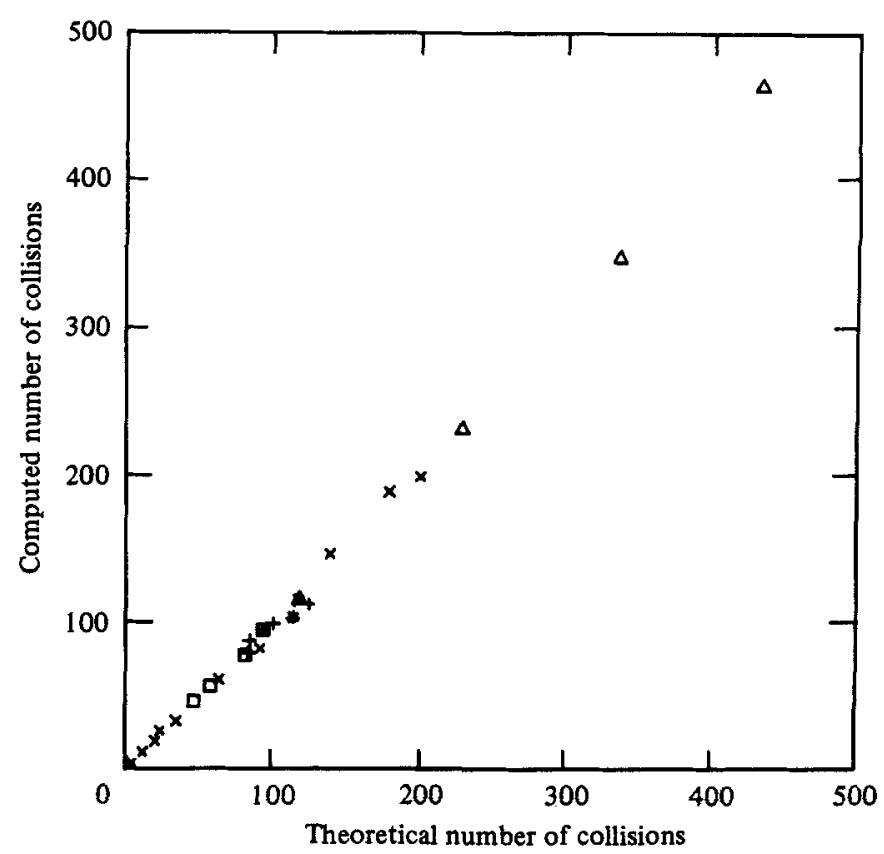

Frgure 18. Comparison of theoretical and computed collision rates : $\times$, laminar shear; $\square$, Brownian motion; $\triangle$, differential sedimentation; + , Brownian motion and laminar shear.

charge. The results suggest that double-layer electrostatic forces determine the onset of coagulation, but, once collisions occur, the coagulation rate depends only on the hydrodynamic and the van der Waals forces. The onset of coagulation is abrupt, and so a quantitative criterion of stability was derived. The combined actions of hydrodynamic and van der Waals forces reduces the collision rate of all particle pairs, but it decreases the collision rate more between particles of similar size. As a result, contrary to the 'non-interacting' system of Part 1, the simulations performed here showed that the size range covered influences the final steady-state size distribution. In Brownian diffusion the rectilinear collision rate increases with the ratio $r_{2} / r_{1}$ $\left(r_{2}>r_{1}\right)$ of the interacting particles; for the 'non-interacting' system of Part 1 this effect is counterbalanced by the relatively small number of large particles. Hydrodynamic and van der Waals forces tend to reduce the collision efficiency relatively more between particles of equal size. Collisions between particles widely different in size therefore become important in determining the evolution of the size distribution. The coagulation process is no longer 'local' in size space, external parameters like the particle size range do become important and so dimensional analysis cannot be used to describe the development of the size distribution. Hunt's (1982) experiments and some of the simulations performed in this study produced size distributions having a slope close to the characteristic $-\frac{3}{2}$ slope suggested by dimensional analysis. However, the detailed investigation of the collision process performed by the computer 'experiment' suggests that quantitative predictions cannot be made.

Adler (1981) computed the collision efficiency for two unequal spheres in simple shear flow under the action of van der Waals attractive forces. For particles very different in size the collision rate is negligible. As a result, the dynamic equilibrium obtained in the simulated population of coagulating particles does not depend on the 
size range considered. The power-law expression for the steady-state size distribution suggested by dimensional analysis is verified in the simulations, but the level of the equilibrium size distribution depends on the relative strength of the shear and the van der Waals energy of attraction.

Simulations for turbulence-induced coagulation were not performed. If interparticle forces are ignored, the analytic estimates for the collision functions suggest that, for particles much smaller than the Kolmogorov microscale, isotropic turbulent shear is equivalent in coagulating power to a rectilinear laminar shear of magnitude 1.72 times the characteristic turbulent strain rate $(\epsilon / \nu)^{\frac{1}{2}}$. Adler's $(1981)$ collision efficiencies than can be used for isotropic turbulent shear induced coagulation. The equivalence with the simple shear is apparent and the same conclusions hold.

Simulations with a non-interacting sedimenting population of particles gave steady-state size distributions in agreement with the theory. Published collision efficiencies derived from theoretical computations assuming Stokes' flow and corrected to be consistent with experimental results (Neiburger et al. 1976) depend both on the relative and the absolute size of the interacting particles. For large particles (larger than about $80 \mu \mathrm{m}$ ) the collision efficiency decreases as the particles become of increasingly different size; for smaller particles collisions between particles both similar and widely different in size are unlikely. Equilibrium size distributions were obtained only in simulations where the smallest particle in suspension was larger than about $15 \mu \mathrm{m}$. The steady-state size distributions attained by the coagulating particles had a slope varying about $-\frac{13}{6}$, which is the slope predicted by dimensional arguments, and depending on the size range considered. Measured size distributions or particles in aerosols (Pruppacher \& Klett 1978, p. 212) and in sewage sludges (Faisst 1976 ) in the size range $10-100 \mu \mathrm{m}$ have a slope varying about $-\frac{13}{6}$. The larger slope of the size distribution has been attributed erroneously in the past to a 'settling' dominated regime where particles settle out of the system. Settling, however, represents a spatially non-homogeneous mass flux (or volume flux, if the particle density is assumed to remain constant after coalescence) which cannot be sustained unless another mechanism operates simultaneously to input mass into the volume of fluid under consideration. The results of the computer simulation help to explain both the steeper slopes of the particle size distributions observed and their variability.

In conclusion, the results of the simulations suggest that a dynamic equilibrium, sustained by the flux of mass through the size spectrum, exists, but a power-law expression of the form predicted by Hunt and Friedlander can be expected only in the shear-induced coagulation regime. The limited size range covered by the simulations did not allow confirmation or otherwise of the hypothesis that different collision mechanisms act independently over separate regions of the size spectrum. The functional dependence of the collision efficiency on the relative size of the sedimenting particles suggest that differential-settling-induced coagulation does not influence the small end of the size spectrum; and Brownian motion is too weak as a coagulating mechanism to affect large particles. To elucidate this point further, information is needed on the influence of hydrodynamic, van der Waals' and electrostatic forces on the collision probability of two particles when two or more of the collision mechanisms examined here act simultaneously.

The simulation described here can also be used to give insight into the spatial fluctuations in particle number and size which occur in a real system. Such information cannot be obtained from the numerical solution of the general dynamic equation, which is a deterministic phenomenological equation and describes the 
behaviour of the suspension averaged over some volume of fluid. Furthermore, there is a good reason to question the suitability of the general dynamic equation to describe the evolution of a coagulating suspension. The general dynamic equation assumes a completely mixed system and ignores correlations between the particles induced by the coagulation process. For example, as particles of a given size in a region of fluid coagulate, a local reduction in their number occurs, so fewer particles of this size remain for further coalescence. If the suspension of particles is poorly mixed or the number of particles is small, then the average behaviour of the suspension predicted by the general dynamic equation may not represent the true average of the local coalescence processes. Gillespie (1972) and Bayewitz et al. (1974) developed the full stochastic equation of the coalescence process and showed that the solution obtained from the general dynamic equation approaches the true stochastic average provided that certain correlations are neglected and that coagulation between particles of equal size is unimportant. The computer model developed in Part 1 is a direct simulation of the processes of collision and coalescence of particles, and, as such, it accounts for all correlations between particle properties. It does not only predict the average spectrum, but it also gives information on higher-order moments of properties of the suspension. This is important since the size distribution predicted by the general dynamic equation will be valid when the standard deviation of the various properties of the suspension is a small fraction of the mean. The Monte Carlo simulation thus provides a unique tool to evaluate the validity of the general dynamic equation to describe the dynamics of a coagulating population of particles and such work is in progress. The small number of particles that are employed in the simulation restricts its application to small regions of the fluid. However, since the coagulation process is mainly local, this may not be a serious defect. Ensemble averages over repeated runs can then represent the true stochastic average of the coagulation process in a larger fluid volume.

The work described herein is taken, in most part, from the Ph.D thesis of I. A. Valioulis submitted to the California Institute of Technology in March 1983. The authors gratefully acknowledge the support of the Office of Ocean Assessment N0AA (NA80RA-D0-0054 and NA82RAD00004) and Sea Grant N0AA (NA 80RAA-D-00120).

\section{REFERENCES}

Aduer, P. M. 1981 Heterocoagulation in shear flow. J. Coll. Interf. Sci. 83, 106-115.

ARP, P. A. \& Mason, S. G. 1976 Orthokinetic collisions of hard spheres in simple shear flow. Can. J. Chem. 54, 3769-3774.

Batchelor, G. K. 1976 Brownian diffusion of particles with hydrodynamic interactions. J. Fluid Mech. 74, 1-29.

BATCHELOR, G. K. \& GREEN, J. T. 1972 The hydrodynamic interactions of two small freely-moving spheres in a linear flow field. J. Fluid Mech. 56, 375-400.

Beyewitz, M. H., Yershalmi, J., Katz, S. \& Shinnar, R. 1974 The extent of correlations in a stochastic coalescence process. J. Atmos. Sci. 31, 1604-1614.

Einstein, A. 1926 The Theory of the Brownian Movement. Dover.

FaIssT, W. K. 1980 Characterization of particles in digested sewage sludge. In Particulates in Water: Characterization, Fate, Effects and Removal (ed. M. C. Kavanagh and J. O. Leckie). Adv. in Chem. Series 189, American Chemical Society.

Findeisen, W. 1939 Zur Frage der Regentröpfenbildung in reinem Wasserwolken. Meteor. Z. 56, $365-368$. 
Frens, G. \& Overbeek, J. T. G. 1971 Repeptization and the theory of elecrostatic colloids. J. Coll. Interf. Sci. 38, 376-387.

Friedlander, S. K. $1960 a$ On the particle size spectrum of atmospheric aerosols. J. Meteorol. 17 , 373-374.

Friedlander, S. K. $1960 b$ Similarity considerations for the particle-size spectrum of a coagulating, sedimenting aerosol. J. Meteorol. 17, 479-483.

Fuchs, N. A. 1964 The Mechanics of Aerosols. Pergamon.

Gillespie, D. T. 1972 The stochastic coalescence model for cloud droplet growth. J. Atmos. Sci. 29, $1496-1510$.

Honig, E. P. \& Mull, P. M. 1971 Tables and equations of the diffuse double layer repulsion at constant potential and at constant charge. J. Coll. Interf. Sci. 36, 258-272.

Honig, E. P., Roebersen, G. J. \& Wiersema, P. H. 1971 Effect of hydrodynamic interaction on the coagulation rate of hydrophobic colloids. J. Coll. Interf. Sci. 36, 97-109.

HunT, J. R. 1982 Self-similar particle-size distributions during coagulation: theory and experimental verification. J. Fluid Mech. 122, 169-185.

JEFFREY, D. J. 1981 Quasi-stationary approximations for the size distribution of aerosols. $J$. Atmos. Sci. 38, 2440-2443.

Monin, A. S. \& YAGLOM, A. M. 1975 Statistical Fluid Mechanics, vol. 2. MIT Press.

Neiburger, M., Lee, I. Y., Lobl, E. \& Rodriguez, L. 1976 Computed collision efficiencies and experimental collection efficiencies on cloud drops. In Proc. Conf. on Cloud Phys. of the Am. Meteor. Soc., Tuscon, Ariz., pp. 73-78.

Pearson, H. J., Valioulis, I. A. \& List, E. J. 1984 Monte Carlo simulation of coagulation in discrete particle-size distributions. Part 1. Brownian motion and fluid shearing. J. Fluid Mech. 143, 367-385.

Pruppacher, H. R. \& Klett, J. D. 1978 Microphysics of Clouds and Precipitation. Reidel.

Saffman, P. G. \& Turner, J. S. 1956 On the collision of drops in turbulent clouds. J. Fluid Mech. 1, 16-30.

Schenkel, J. H. \& Kitchener, J. A. 1960 A test of the Derjaguin-Verwey-Overbeek theory with a colloidal suspension. Trans. Faraday Soc. 56, 161-173.

Sмolvchowski, M. 1916 Drei Vorträge über Diffusion, brownsche Bewegung und Koagulation von Kolloidteilchen. Phys. Z. 17, 557-585.

Smoluchowski, M. 1917 Versuch einer mathematischen Theorie der Koagulationskinetic kolloider Lösungen. Z. Phys. Chem. 92, 129.

Spielman, A. L. 1970 Viscous interactions in Brownian coagulation. J. Coll. Interf. Sci. 33, $562-571$.

Stimson, M. \& Jeffrey, G. B. 1926 The motion of two spheres in a viscous fluid. Proc. R. Soc. Lond. A 111, 110-116.

TAG, P. M. 1976 The effect of utilizing empirically derived values of coalescence efficiency in a microphysical cloud model. In Proc. Conf. on Cloud Phys. of the Am. Meteor. Soc., Tuscon, Ariz., pp. 73-78.

VALIoulis, I. A. \& List, E. J. 1983 Collision efficiencies of diffusing spherical particles: Hydrodynamic, van der Waals' and electrostatic forces. Adv. Coll. Interface Sci. (in press).

Zeichner, G. R. \& Schowalter, W. R. 1977 Use of trajectory analysis to study the stability of colloidal dispersions in flow fields. AIChE J. 23, 243-254. 